\title{
The normal zeta function of the free class two nilpotent group on four generators
}

\author{
Pirita Maria Paajanen \\ Mathematical Institute \\ 24-29 St Giles' \\ Oxford OX1 3LB \\ UK \\ paajanen@maths.ox.ac.uk
}

14th November 2018

\begin{abstract}
We calculate explicitly the normal zeta function of the free group of class two on four generators, denoted by $F_{2,4}$. This has Hirsch length ten.
\end{abstract}

\section{Introduction}

A zeta function of a group is a tool used in infinite group theory to understand subgroup growth, the study of the properties of the sequence $\left(a_{n}(G)\right)_{n \in \mathbb{N}}$, where $a_{n}(G)$ encodes the number of subgroups of index $n$ in a finitely generated infinite group $G$. By studying analytic properties of the zeta function

$$
\zeta_{\bar{G}}^{\leq}(s)=\sum_{n=1}^{\infty} a_{n}^{\leq}(G) n^{-s},
$$

where

$$
a_{n}^{\leq}(G)=|\{H \leq G:|G: H|=n\}|,
$$

we hope to get algebraic information about the group.

We can define a normal zeta function

$$
\zeta_{G}^{\triangleleft}(s)=\sum_{n=1}^{\infty} a_{n}^{\triangleleft}(G) n^{-s}
$$


by the additional condition that we count only normal subgroups of $G$, so

$$
a_{n}^{\triangleleft}(G)=|\{H \triangleleft G:|G: H|=n\}| .
$$

In the example in this paper we calculate a zeta function of a finitely generated torsion-free nilpotent group — we call these the $\mathfrak{T}$-groups - and the nilpotency gives us the extra feature that for $* \in\{\triangleleft, \leq\}$ the zeta function decomposes as an Euler product of local factors (also known as the local zeta functions) over primes $p$

$$
\zeta_{G}^{*}(s)=\prod_{p} \zeta_{G, p}^{*}(s)
$$

where

$$
\zeta_{G, p}^{*}(s)=\sum_{n=0}^{\infty} a_{p^{n}}^{*}(G) p^{-n s} .
$$

One of the main results in [6] was to prove that these local zeta functions are rational functions in $p^{-s}$. Moreover, in [6] it is proved that the zeta functions of the free nilpotent groups of class two are uniform; this means that for almost all, that is, for all but finitely many, primes $p$ the local factor is the same for each prime. It should be noted that this is not always the case, there are examples of zeta functions whose local factors don't fall into even finitely many classes for primes $p$. The elliptic curve example by du Sautoy discussed in 1 and 3] explains why the the local factors of a zeta function depend on the points on an elliptic curve modulo $p$.

More generally, in [5] du Sautoy and Grunewald link the theory of zeta functions of groups to the classical problem of counting points on varieties.

Theorem 1.1 (du Sautoy, Grunewald). Let $G$ be a finitely generated nilpotent infinite group. Then there exist an algebraic variety $Y$ defined over $\mathbb{Q}$, consisting of finitely many irreducible components $V_{i}, i \in T$, defined over $\mathbb{Q}$. All these irreducible components are smooth and intersect normally. There also exist rational functions $P_{I}(x, y) \in \mathbb{Q}(x, y)$ for each $I \subseteq T$ with the property that for almost all primes $p$

$$
\zeta_{G, p}^{*}(s)=\sum_{I \subseteq T} d_{I, p} P_{I}\left(p, p^{-s}\right)
$$

where

$$
d_{I, p}=\left|\left\{a \in \bar{Y}\left(\mathbb{F}_{p}\right): a \in \bar{V}_{i} \Leftrightarrow i \in I\right\}\right|
$$

and $\bar{Y}$ means the reduction $\bmod p$ of the variety $Y$. 
However this theorem still has mainly theoretical meaning. One of the big questions recently has been to describe which varieties appear in the decomposition. In [1] and [3] we saw that a zeta function of a certain group depends on the number of $\mathbb{F}_{p}$-points on an elliptic curve. In this paper we can add Fano varieties of linear spaces to the list of varieties we need to consider in this context. But even so, we need to be very careful, as we shall see in this example. Not all points on the Fano variety of planes appear in the zeta function. Only one of the two rulings of planes gets picked up by the zeta function.

In some sense this work can also be considered as a generalisation of Voll's work in 13 and 12 . In the paper 13 , he proves that a local normal zeta function satisfies a functional equation of the form

$$
\left.\zeta_{G, p}^{\triangleleft}(s)\right|_{p \mapsto p^{-1}}=(-1)^{l} p^{m-n s} \cdot \zeta_{G, p}^{\triangleleft}(s),
$$

for some $l, m, n \in \mathbb{N}$, if the Pfaffian hypersurface associated to the group is smooth, absolutely irreducible and does not contain lines. The example that we shall present in this paper however involves a group whose associated Pfaffian is smooth, contains lines and planes, and still satisfies a functional equation of the same form.

Apart from the pure interest in such a standard example as a nilpotent free group, this calculation has application to the problem of counting $p$ groups. As du Sautoy explains in [2], counting normal subgroups in the free class $c$ nilpotent groups on $d$ generators is intimately related to counting $p$ groups of class $c$ on $d$ generators - the latter being identifiable by quotients of the former.

In addition to giving an explicit calculation of the normal zeta function of $F_{2,4}$, we shall do it in a systematic and theoretical manner which hopefully leads to a better theoretical understanding about the shape of the function and its poles. In a future paper [10] I shall consider the more general case, where the Pfaffian is assumed to be smooth.

This result was already announced in [13].

It also should be noted that no computer calculations are used in this example. If one tried to calculate this example using $p$-adic integrals, the cone conditions would contain 53 polynomial equations and involve 55 variables. Thanks are due to Luke Woodward who generated these conditions for me.

\section{$1.1 \quad$ Results}

Let $F_{2, d}$ be the free class two, $d$ generator nilpotent group. $F_{2, d}=F_{d} / \gamma_{3}\left(F_{d}\right)$, where $F_{d}$ is the free group on $d$ generators and $\gamma_{3}\left(F_{d}\right)$ is the third term in 
the lower central series of $F_{d} . F_{2, d}$ has a presentation

$F_{2, d}=\left\langle x_{1}, \ldots, x_{d}, y_{1}, \ldots, y_{\frac{d(d-1)}{2}}:\left[x_{1}, x_{2}\right]=y_{1},\left[x_{1}, x_{3}\right]=y_{2}, \ldots,\left[x_{d-1}, x_{d}\right]=y_{\frac{d(d-1)}{2}}\right\rangle$

with the convention that generators not appearing explicitly in the relations are assumed to commute. Note that for this group $\left[F_{2, d}, F_{2, d}\right]=Z\left(F_{2, d}\right)$.

To start with, let us recall from [6] what the normal zeta functions of smaller examples of the class two nilpotent free groups look like. The two generator one is isomorphic to the Heisenberg group and has local normal zeta function

$$
\zeta_{F_{2,2}, p}^{\triangleleft}(s)=\frac{1}{\left(1-p^{-s}\right)\left(1-p^{1-s}\right)\left(1-p^{2-3 s}\right)} .
$$

The global zeta function $\zeta_{F_{2,2}}^{\triangleleft}(s)=\prod_{p} \zeta_{F_{2,2}, p}^{\triangleleft}(s)$ has abscissa of convergence 2. The normal zeta function of $F_{2,3}$ is

$$
\zeta_{F_{2,3}, p}^{\triangleleft}(s)=\frac{1+p^{3-3 s}+p^{4-3 s}+p^{6-5 s}+p^{7-5 s}+p^{10-8 s}}{\left(1-p^{-s}\right)\left(1-p^{1-s}\right)\left(1-p^{2-s}\right)\left(1-p^{5-3 s}\right)\left(1-p^{8-5 s}\right)\left(1-p^{9-6 s}\right)} .
$$

The global zeta function has abscissa of convergence 3 .

In order to write the normal zeta function of $F_{2,4}$ in a reasonable form an explicit expression would take two and half pages of polynomial formula - we need to introduce some notation and terminology. We shall get back to these definitions later and terminology will be made more precise.

Definition 1.2. The Igusa factor with $n$ variables $\mathbf{U}=\left(U_{1}, \ldots, U_{n}\right)$ is defined to be

$$
I_{n}(\mathbf{U})=\sum_{I \subseteq\{1, \ldots, n\}} b_{I}\left(p^{-1}\right) \prod_{i \in I} \frac{U_{i}}{1-U_{i}},
$$

where $b_{I}(p)$ is the number of $\mathbb{F}_{p}$-points on a flag variety of type $I$, this is a polynomial in $p$, which can be written in terms of $p$-binomial coefficients and is independent of the prime $p$.

The precise definition of a flag variety of type $I$ will be given in 2.2 ,

Example 1.3. In the above, we can write

$$
\zeta_{F_{2,3}, p}^{\triangleleft}(s)=\zeta_{\mathbb{Z}^{3}, p}(s) \zeta_{p}(6 s-9) \cdot I_{2}\left(U_{1}, U_{2}\right),
$$

where $U_{1}=p^{8-5 s}$ and $U_{2}=p^{5-3 s}$, and $\zeta_{p}(s)=\frac{1}{1-p^{-s}}$ denotes a local factor of the Riemann zeta function. 
Let us denote by $d=h\left(F_{2,4}^{a b}\right)=4$ the torsion-free rank of the abelianisation and by $d^{\prime}=h\left(Z\left(F_{2,4}\right)\right)=6$ the torsion-free rank of the centre, which is also an abelian group. In the general case of free class two nilpotent groups $d$ will be number of generators and $d^{\prime}=\frac{d(d-1)}{2}$.

As will be explained later, to each class two nilpotent group we can naturally associate a Pfaffian hypersurface in $\mathbb{P}^{d^{\prime}-1}$. Let $d_{i}$ be the dimension and $c_{i}$ the codimension of the Fano variety of the $(i-1)$-dimensional linear subspaces on the Pfaffian. Then $n_{i}=c_{i}+d_{i}$ is the dimension of the variety of $(i-1)$-dimensional linear subspaces in $\mathbb{P}^{d^{\prime}-1}$.

Definition 1.4. We call a rational function of the form

$$
E_{i}\left(X_{i}, Y_{i}\right)=\frac{p^{-d_{i}} Y_{i}-p^{-n_{i}} X_{i}}{\left(1-X_{i}\right)\left(1-Y_{i}\right)}
$$

the $i^{\text {th }}$ exceptional factor with variables $X_{i}$ and $Y_{i}$.

With the above definitions we can now write down an expression for the zeta function of $F_{2,4}$. It does show some structure, which would be completely missing if the zeta function were to be written explicitly.

Theorem 1.5. The local normal zeta function of $F_{2,4}$ is for (almost) all primes $p$

$\zeta_{F_{2,4}, p}^{\triangleleft}(s)=\zeta_{\mathbb{Z}^{4}, p}(s) \zeta_{p}(10 s-24) \cdot\left(W_{0}\left(p, p^{-s}\right)+\mathfrak{n}_{1}(p) W_{1}\left(p, p^{-s}\right)+\mathfrak{n}_{2}(p) W_{2}\left(p, p^{-s}\right)+\mathfrak{n}_{3}(p) W_{3}\left(p, p^{-s}\right)\right)$

where

$$
\begin{aligned}
& \mathfrak{n}_{1}(p)=\left(p^{2}+1\right)\left(p^{2}+p+1\right), \\
& \mathfrak{n}_{2}(p)=(p+1)\left(p^{2}+1\right)\left(p^{2}+p+1\right), \\
& \mathfrak{n}_{3}(p)=\left(p^{2}+1\right)(p+1),
\end{aligned}
$$

and

$$
W_{i}(X, Y)=I_{5-i}\left(X_{5}, \ldots, X_{i+1}\right) E_{i}\left(X_{i}, Y_{i}\right) I_{i-1}\left(Y_{i-1}, \ldots, Y_{1}\right)
$$

for $i=0,1,2,3$, with the convention that $E_{0}=I_{-1}=I_{0}=1$. The numerical data are

$$
\begin{aligned}
& X_{i}=p^{i(10-i)-(4+i) s} \\
& Y_{1}=p^{8-3 s} \\
& Y_{2}=p^{13-5 s} \\
& Y_{3}=p^{15-6 s} .
\end{aligned}
$$


One can calculate the closed expression of this zeta function where everything is put over a common denominator. However, it is not done here, since the numerator is a polynomial with 490 terms and the whole zeta function would take more than two pages to write out. We can read off the degree of polynomial subgroup growth from the expression above.

Corollary 1.6. The abscissa of convergence of the global zeta function is 4 .

This is still the torsion-free rank of the abelianisation. However, for $F_{2,5}$ the abscissa is bigger than 5 , which can be deduced for instance from Theorem 1.3 in 9 .

Corollary 1.7. The function satisfies a functional equation of the form

$$
\left.\zeta_{F_{2,4}, p}^{\triangleleft}(s)\right|_{p \mapsto p^{-1}}=p^{45-14 s} \cdot \zeta_{F_{2,4}, p}^{\triangleleft}(s) .
$$

This is of the form conjectured to hold for all class two normal zeta functions, see section 5 .

Acknowledgements. I would like to thank Christopher Voll for numerous helpful conversations and Marcus du Sautoy for his encouragement as a supervisor. My DPhil, part of which this paper forms, has been financially supported by Academy of Finland, Finnish Academy of Science and Letters, Osk. Huttunen Foundation and Oxford University Graduate Scholarship Scheme. I am also grateful for the friendliness of Helsinki University of Technology during the writing up of this paper.

\section{Theory behind the calculation}

The class two nilpotent groups split naturally into two parts, the derived group and the abelianisation. With free class two groups we even have $G^{\prime}=$ $[G, G]=Z(G)$. By the Mal'cev correspondence there is a Lie algebra $=(G)$ over $\mathbb{Z}$ constructed as the image under log of $G$.

For a Lie algebra, and $* \in\{\leq, \triangleleft\}$, we define the zeta function

$$
\zeta^{*}(s)=\sum_{n=1}^{\infty} a_{n}^{*}() n^{-s}
$$

where $a_{n}^{*}()$ is the number of subalgebras or ideals of of index $n$. Then we have the following theorem 
Theorem 2.1 ([6]). Let $G$ be a finitely generated nilpotent group. For $* \in$ $\{\leq, \triangleleft\}$ and almost all primes $p$

$$
\zeta_{G, p}^{*}(s)=\zeta_{(G), p}^{*}(s) .
$$

This allows us to concentrate on counting in a ring setting, which is usually easier than the corresponding group setting.

According to [6] (Lemma 6.1) for class two normal zeta functions it is enough to run over the lattices of the centre of the group (or Lie algebra) in order to calculate the zeta function. In particular, if for each lattice $\Lambda^{\prime} \subseteq^{\prime}$ we define $X\left(\Lambda^{\prime}\right) / \Lambda^{\prime}:=Z\left(/ \Lambda^{\prime}\right)$, then

$$
\zeta^{\triangleleft}(s)=\left.\zeta_{\mathbb{Z}^{d}}(s) \sum_{\Lambda^{\prime} \subseteq^{\prime}}\right|^{\prime}:\left.\Lambda^{\prime}\right|^{d-s}\left|: X\left(\Lambda^{\prime}\right)\right|^{-s}
$$

We can also use the local version of this lemma by considering ${ }_{p}=\otimes_{\mathbb{Z}} \mathbb{Z}_{p}$. An ideal of a finite index in ${ }_{p}$ corresponds to an ideal of a $p$-power index in . We shall call a lattice $\Lambda^{\prime} \subseteq \mathbb{Z}_{p}^{d^{\prime}}$ maximal if $p^{-1} \Lambda^{\prime} \nsubseteq \mathbb{Z}_{p}^{d^{\prime}}$. It is enough to consider only maximal lattices of $p$-power index, since if $\Lambda^{\prime}$ is not maximal, then in general, $\Lambda^{\prime}=p^{r_{d^{\prime}}} \Lambda_{\max }^{\prime}$, where $\Lambda_{\max }^{\prime}$ is maximal in its class. Now

$$
\left.\right|_{p} ^{\prime}: \Lambda^{\prime}\left|=p^{d^{\prime} r_{d^{\prime}}}\right|_{p}^{\prime}: \Lambda_{\max }^{\prime} \mid
$$

and

$$
\left.\right|_{p}: X\left(\Lambda^{\prime}\right)\left|=p^{d r_{d^{\prime}}}\right|_{p}: X\left(\Lambda_{\text {max }}^{\prime}\right) \mid .
$$

Then we have

$$
\begin{aligned}
\zeta_{, p}^{\triangleleft}(s)=\zeta_{p}^{\triangleleft}(s) & =\left.\zeta_{\mathbb{Z}_{p}^{d}}(s) \sum_{\Lambda^{\prime} \subseteq_{p}^{\prime}}\right|_{p} ^{\prime}:\left.\left.\Lambda^{\prime}\right|^{d-s}\right|_{p}:\left.X\left(\Lambda^{\prime}\right)\right|^{-s} \\
& =\zeta_{\mathbb{Z}_{p}^{d}}(s) \zeta_{p}\left(\left(d+d^{\prime}\right) s-d d^{\prime}\right) A\left(p, p^{-s}\right),
\end{aligned}
$$

where

$$
A\left(p, p^{-s}\right)=\left.\sum_{\substack{\Lambda^{\prime} \complement_{p}^{\prime} \\ \Lambda^{\prime} \text { maximal }}}\right|_{p} ^{\prime}:\left.\left.\Lambda^{\prime}\right|^{d-s}\right|_{p}:\left.X\left(\Lambda^{\prime}\right)\right|^{-s} .
$$

Recall from [12] the definition of the weight functions

$$
\begin{aligned}
w\left(\Lambda^{\prime}\right) & :=\log _{p}\left(\left.\right|_{p} ^{\prime}: \Lambda^{\prime} \mid\right) \\
w^{\prime}\left(\Lambda^{\prime}\right) & :=w\left(\Lambda^{\prime}\right)+\log _{p}\left(\left.\right|_{p}: X\left(\Lambda^{\prime}\right) \mid\right) .
\end{aligned}
$$

Put $T:=p^{-s}$. Then we can write our generating function as

$$
A(p, T)=\sum_{\Lambda^{\prime} \subseteq_{p}^{\prime}, \text { maximal }} p^{d w\left(\Lambda^{\prime}\right)} T^{w^{\prime}\left(\Lambda^{\prime}\right)} .
$$


Let us consider ${ }_{p}$ and a lattice $\Lambda^{\prime} \in{ }_{p}^{\prime} \cong \mathbb{Z}_{p}^{d^{\prime}}$. In order to do the counting effectively, we enumerate the lattices in the centre using the elementary divisor type. The lattices maximal in their class have elementary divisor types $\left(1, p^{r_{d^{\prime}-1}}, p^{r_{d^{\prime}-2}+r_{d^{\prime}-1}}, \ldots, p^{r_{1}+r_{2}+\cdots+r_{d^{\prime}-1}}\right)$, where $r_{i} \geq 0$. Let us denote by $\left\{i_{1}, \ldots, i_{n}\right\}_{<}$the ordered set where $i_{1}<i_{2}<\ldots i_{l}$. Then the type of lattice is given by $\nu\left(\Lambda^{\prime}\right)=\left(I, r_{I}\right)$ where

$$
I=\left\{i_{1}, \ldots i_{l}\right\}_{<} \subseteq\left\{1, \ldots, d^{\prime}-1\right\}, r_{I}=\left(r_{i_{1}}, \ldots, r_{i_{l}}\right)
$$

and $I=\left\{i_{j} \in\left\{1, \ldots, d^{\prime}-1\right\}: r_{i_{j}}>0\right\}$.

This way we have the same enumeration for the lattices, and for the weight functions depending on the lattices' types. From these definitions and the above notation for the lattices we have immediately

$$
w\left(\Lambda^{\prime}\right)=\sum_{i \in I} i r_{i}
$$

Definition 2.2. A flag of type $I$ in $\mathbb{P}^{d^{\prime}-1}\left(\mathbb{F}_{p}\right), I \subseteq\left\{1, \ldots, d^{\prime}-1\right\}$ is a sequence $\left(V_{i_{j}}\right)_{i_{j} \in I}$ of incident vector spaces

$$
\mathbb{P}^{d^{\prime}-1}\left(\mathbb{F}_{p}\right)>V_{i_{1}}>\cdots>V_{i_{l}}>\{0\}
$$

with $\operatorname{codim}_{\mathbb{F}_{p}}\left(V_{i_{j}}\right)=i_{j}-i_{j-1}$ in the vector space $V_{i_{j-1}}$. The flags of type $I$ form a projective variety $\mathcal{F}_{I}$, whose number of $\mathbb{F}_{p}$-points is given by $b_{I}(p) \in \mathbb{Z}[p]$ a polynomial whose leading term equals $p^{\operatorname{dim} \mathcal{F}_{I}}$ and can be easily expressed as a product of $p$-binomial coefficients. Using duality properties of $p$-binomial coefficients we observe that

$$
b_{I}\left(p^{-1}\right)=p^{-\operatorname{dim} \mathcal{F}_{I}} b_{I}(p) .
$$

$\mathrm{SL}_{d^{\prime}}\left(\mathbb{Z}_{p}\right)$ acts transitively on maximal lattices of an elementary divisor type

$$
\left(1, p^{r_{d^{\prime}-1}}, p^{r_{d^{\prime}-2}+r_{d^{\prime}-1}}, \ldots, p^{r_{1}+r_{2}+\cdots+r_{d^{\prime}-1}}\right)
$$

relative to the standard lattice.

Let $G_{\nu}$ denote the stabiliser of the diagonal matrix

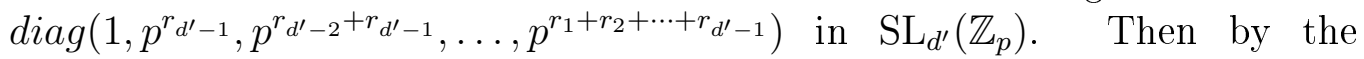
orbit-stabiliser theorem we have

$$
\{\text { maximal lattices of type } \nu\} \stackrel{1-1}{\leftrightarrow} \mathrm{SL}_{d^{\prime}}\left(\mathbb{Z}_{p}\right) / G_{\nu} \text {. }
$$

Thus we identify a maximal lattice $\Lambda^{\prime}$ with the pair $\left(\nu, \alpha G_{\nu}\right)$ consisting of its partition of elementary divisors and the respective coset of the stabiliser $G_{\nu}$. 
Let $\alpha \in \mathrm{SL}_{d^{\prime}}\left(\mathbb{Z}_{p}\right)$ and $I$ be a type of a lattice $\Lambda^{\prime}$. Let $\bar{\alpha}$ denote the reduction $\bmod p$ and define vector spaces for $i_{j} \in I, I=\left\{i_{1}, \ldots, i_{l}\right\}_{<}$,

$$
V_{i_{j}}=\left\langle\overline{\alpha^{i_{1}}}, \ldots, \overline{\alpha^{i_{j}}}\right\rangle<\mathbb{P}^{d^{\prime}-1}\left(\mathbb{F}_{p}\right)
$$

We observe that $\operatorname{codim}_{\mathbb{F}_{p}}\left(V_{i_{j}}\right)=i_{j}-i_{j-1}$.

Following Voll we call the flag $\left(V_{i}\right)_{i \in I}$ of type $I$ the flag associated with $\Lambda^{\prime}$ if $\nu\left(\Lambda^{\prime}\right)=I$ and $\Lambda^{\prime}$ corresponds to the coset $\alpha G_{\nu}$ under the 1-1 correspondence.

Definition 2.3. Given a fixed flag variety $\mathcal{F}_{I}$ of type $I=\left\{i_{1}, \ldots, i_{l}\right\}_{<}$on $\mathbb{P}^{d^{\prime}-1}$, then we call a maximal lattice $\Lambda^{\prime}$ of type $J=\left\{j_{1}, \ldots, j_{k}\right\}_{<}$where $k \geq l$ a lift of $\mathcal{F}_{I}$ if its associated flag $\left(V_{j}\right)_{j \in J}$ contains $\mathcal{F}_{I}, I \subseteq J$ and $i_{m}=j_{m}$ for $m=1, \ldots, l$.

The reason we define these is that we can describe $\left.\right|_{p}: X\left(\Lambda^{\prime}\right) \mid$ by set of polynomial equations.

Theorem 2.4 (Voll [13]). Let $\Lambda^{\prime}$ correspond to the coset $\alpha G_{\nu}$ under the correspondence defined above, where $\alpha \in \mathrm{SL}_{6}\left(\mathbb{Z}_{p}\right)$ with column vectors $\alpha^{j}$, $j=1, \ldots, 6$. Then $\left.\right|_{p}: X\left(\Lambda^{\prime}\right) \mid$ equals the index of the kernel of the following system of linear congruences in ${ }_{p} /_{p}^{\prime}$ :

$$
\forall i \in\{1, \ldots, 6\} \quad \overline{\mathbf{g}} M\left(\alpha^{j}\right) \equiv 0 \quad \bmod p^{r_{i}+\cdots+r_{6}}
$$

where $\overline{\mathbf{g}}=\left(\overline{g_{1}}, \ldots, \overline{g_{4}}\right) \in{ }_{p} /{ }_{p}^{\prime} \cong \mathbb{Z}_{p}^{4}$.

From this it is clear that we need to separate cases when $M\left(\alpha^{j}\right)$ is singular and when it is not. The space of singular matrices can be described using the Pfaffian hypersurface, which is defined by the vanishing of the determinant of the matrix of relations $\operatorname{det}(M(\mathbf{y}))=0$.

In addition to the above two weight functions we need to define the multiplicity function $\mu$ which measures how many lattices there are of each given type.

Definition 2.5. Let $\Lambda^{\prime}$ be a lattice of type $I$. The multiplicity of $\Lambda^{\prime}$, denoted by $\mu\left(\Lambda^{\prime}\right)$ is the number of lattices of type $I$ divided by the number of points on the flag variety of type $I$.

We can calculate the multiplicity $\mu\left(\Lambda^{\prime}\right)$ by defining the function mu (as a 'measuret' or a multiplicity) to measure the size of the set of $x \in p \mathbb{Z}_{p} /\left(p^{a}\right)$ of a fixed $p$-adic valuation as follows: 
Definition 2.6. Let $a, b$ be positive integers. We define a function $\mu$ of $a$ and $b$ as

$$
\mu(a, b)=\left|\left\{x \in p \mathbb{Z}_{p} /\left(p^{a}\right): v_{p}(x)=b\right\}\right|= \begin{cases}1 & \text { if } a=b \\ p^{a-b}\left(1-p^{-1}\right) & \text { if } a>b \\ 0 & \text { otherwise }\end{cases}
$$

This definition extends naturally also to a vector $\mathbf{b}=\left(b_{1}, \ldots, b_{n}\right)$. We denote $\mu(a, \mathbf{b})=\left|\left\{\mathbf{x} \in p \mathbb{Z}_{p} /\left(p^{a}\right): v_{p}\left(x_{i}\right)=b_{i}\right\}\right|=\mu\left(a ; b_{1}, b_{2}, b_{3}, \ldots, b_{n}\right)=$ $\mu\left(a, b_{1}\right) \mu\left(a, b_{2}\right) \ldots \mu\left(a, b_{n}\right)$.

Now we can calculate the multiplicity of lattices of a given type $I$.

Lemma 2.7. Let $\Lambda^{\prime}$ be a lattice of type $I$ corresponding to the coset $\alpha G_{\nu}$ under the 1-1 correspondence. Let $a_{i j}$ be the ij-entry of $\alpha$ (in the ith column from the right and (j-1)th row from the bottom). Then the multiplicity of $\Lambda^{\prime}$ is

$$
\mu\left(\Lambda^{\prime}\right)=\prod_{\substack{i \in I \\ j \in I \\ j \leq i}} \sum_{a_{i j}=1}^{\sum_{k=i}^{j} r_{k}} \mu\left(\sum_{k=i}^{j} r_{k}, a_{i j}\right)=p^{-\operatorname{dim} \mathcal{F}_{I}} p^{\sum_{i \in I}\left(d^{\prime}-i\right) i r_{i}} .
$$

Proof. A simple computation using the orbit-stabiliser theorem.

\section{The group and its Pfaffian hypersurface}

The free class two nilpotent group on 4 generators has the following presentation

$$
\begin{gathered}
F_{2,4}=\left\langle x_{1}, x_{2}, x_{3}, x_{4}, y_{1}, y_{2}, y_{3}, y_{4}, y_{5}, y_{6}:\left[x_{1}, x_{2}\right]=y_{1},\left[x_{1}, x_{3}\right]=y_{2},\right. \\
\left.\left[x_{1}, x_{4}\right]=y_{3},\left[x_{2}, x_{3}\right]=y_{4},\left[x_{2}, x_{4}\right]=y_{5},\left[x_{3}, x_{4}\right]=y_{6}\right\rangle .
\end{gathered}
$$

We can also write the commutator structure in the form of the matrix of relations as

$$
M(\mathbf{y})=\left(\begin{array}{cccc}
0 & y_{1} & y_{2} & y_{3} \\
-y_{1} & 0 & y_{4} & y_{5} \\
-y_{2} & -y_{4} & 0 & y_{6} \\
-y_{3} & -y_{5} & -y_{6} & 0
\end{array}\right)
$$

Then the presentation is given by

$$
F_{2,4}=\left\langle x_{1}, x_{2}, x_{3}, x_{4}, y_{1}, y_{2}, y_{3}, y_{4}, y_{5}, y_{6}:\left[x_{i}, x_{j}\right]=M(\mathbf{y})_{i j}\right\rangle
$$


The Pfaffian hypersurface is defined by the square-root of the determinant of the matrix of relations $M(\mathbf{y})$; in this case it is defined by the equation

$$
y_{1} y_{6}-y_{2} y_{5}+y_{3} y_{4}=0 \text {. }
$$

This is a smooth quadric four-fold in $\mathbb{P}^{5}$. It is a fact (see e.g. Theorem 22.13 in [7]) that a smooth quadric of dimension $m$ contains linear subspaces only up to dimension less than or equal to $m / 2$, thus the linear subspaces lying on the Pfaffian hypersurface $\mathfrak{P}$ associated with $F_{2,4}$ are points, lines and planes, these are also smooth by the above mentioned Theorem 22.13 . As we can find, for example from from [8] p. 5, there are $\mathfrak{P}_{1}(p)=\left(p^{2}+1\right)\left(p^{2}+p+1\right)$ points, $\mathfrak{P}_{2}(p)=(p+1)\left(p^{2}+1\right)\left(p^{2}+p+1\right)$ lines and $\mathfrak{P}_{3}(p)=2\left(p^{2}+1\right)(p+1)$ planes over $\mathbb{F}_{p}$. There are two rulings of planes, which again is from the general theory of quadrics, see Theorem 22.14 in [7].

Thus the different lifts of fixed flags that lie on the Pfaffian hypersurface (Recall Definition 2.3) and can cause different congruence conditions and weight functions are the following:

1. The lattice $\Lambda^{\prime}$ lifts the flag $\left\langle\alpha^{1}\right\rangle$, which consists of a point on the Pfaffian. Note that the line $\left\langle\alpha^{1}, \alpha^{2}\right\rangle$ does not lie on the quadric four-fold in this case.

2. (a) The lattice $\Lambda^{\prime}$ lifts the flag $\left\langle\alpha^{1}, \alpha^{2}\right\rangle$, which consists of a line on the Pfaffian.

(b) $\Lambda^{\prime}$ lifts the flag $\left\langle\alpha^{1}, \alpha^{2}\right\rangle \geq\left\langle\alpha^{1}\right\rangle$, which is a line-point pair on the Pfaffian.

3. (a) $\Lambda^{\prime}$ lifts the flag $\left\langle\alpha^{1}, \alpha^{2}, \alpha^{3}\right\rangle$, which consists of a plane on the Pfaffian.

(b) $\Lambda^{\prime}$ lifts the flag $\left\langle\alpha^{1}, \alpha^{2}, \alpha^{3}\right\rangle \geq\left\langle\alpha^{1}, \alpha^{2}\right\rangle$, which consists of a planeline pair on the Pfaffian.

(c) $\Lambda^{\prime}$ lifts the flag $\left\langle\alpha^{1}, \alpha^{2}, \alpha^{3}\right\rangle \geq\left\langle\alpha^{1}\right\rangle$, consisting of a plane-point pair on the Pfaffian.

(d) $\Lambda^{\prime}$ lifts the flag $\left\langle\alpha^{1}, \alpha^{2}, \alpha^{3}\right\rangle \geq\left\langle\alpha^{1}, \alpha^{2}\right\rangle \geq\left\langle\alpha^{1}\right\rangle$, which is a fixed plane-line-point triplet on the Pfaffian.

\subsection{Weight functions}

In this section we consider different types of lattices and what kind of weight functions $w^{\prime}\left(\Lambda^{\prime}\right)$ we obtain following the above geometric list. 
The easiest case is when a lattice lifts a flag no part of which lies on the Pfaffian hypersurface, so all the matrices $M\left(\alpha^{i}\right)$ are non-singular and the congruence conditions reduce to:

$$
\forall i \in\{1, \ldots, 6\} \quad \overline{\mathbf{g}} \equiv 0 \quad \bmod p^{r_{i}+\cdots+r_{5}} .
$$

The weight function $w^{\prime}\left(\Lambda^{\prime}\right)=\sum_{i \in I}(4+i) r_{i}$ depends only on the lattice's type.

Now we need to consider the conditions on the Pfaffian as listed above. The easiest of them is that a lattice $\Lambda^{\prime}$ lifts a fixed point $x \in \mathbb{P}^{5}\left(\mathbb{F}_{p}\right)$ on the Pfaffian hypersurface.

Lemma 3.1 (Voll, Proposition 4 in [13]). Let $x \in \mathfrak{P}$, and let $\Lambda^{\prime}$ be a lattice lifting this fixed point. Then the weight function is $w^{\prime}\left(\Lambda^{\prime}\right)=\sum_{i \in I}(4+$ i) $r_{i}-2 \min \left\{r_{1}, v_{p}\left(a_{11}\right)\right\}$. This depends on more than the lattice's type, but it is independent of the point $x$ chosen.

Proof. (Proposition 4 in [13])

Let $\alpha^{1}=\left(a_{11}, a_{12}, \ldots, a_{16}\right) \in \mathbb{P}^{5}\left(\mathbb{Z}_{p} / p^{r_{1}}\right)$ and $\alpha^{1}=(0,1, \ldots, 1) \bmod p$. Then we can choose local coordinates such that around any of the $\mathfrak{n}_{1}(p)$ points of the Pfaffian hypersurface $\bmod p$ the congruence conditions look like

$$
\begin{array}{r}
\mathbf{g} \cdot\left(\begin{array}{cccc}
0 & a_{11} & 0 & 0 \\
-a_{11} & 0 & 0 & 0 \\
0 & 0 & 0 & 1 \\
0 & 0 & -1 & 0
\end{array}\right) \\
\quad \overline{\mathbf{g}} \equiv 0 \quad \bmod p^{r_{2}+\cdots+r_{5}} .
\end{array}
$$

It can be read off that the weight function is $w^{\prime}\left(\Lambda^{\prime}\right)=\sum_{i \in I}(4+i) r_{i}-$ $2 \min \left\{r_{1}, v_{p}\left(a_{11}\right)\right\}$.

With lines we have the following situation: The variety of lines is smooth and irreducible, and all lines belong to the same family, so it is enough to consider one given line only.

Lemma 3.2. The weight function for lattices lifting a given line on the Pfaffian is $w^{\prime}\left(\Lambda^{\prime}\right)=6 r_{2}-\min \left\{r_{2}, v_{p}\left(a_{15}\right), v_{p}\left(a_{25}\right), v_{p}\left(a_{24}\right)\right\}$. This is independent of the line chosen.

Proof. The lattice of type $\left(1,1,1,1, p^{r_{2}}, p^{r_{2}}\right)$ is in one-to-one correspondence with the pair of vectors $\alpha^{1}=\left(a_{15}, a_{14}, a_{13}, a_{12}, 0,1\right)^{t}$ and $\alpha^{2}=$ 
$\left(a_{25}, a_{24}, a_{23}, a_{22}, 1,0\right)^{t}$, where $a_{i j} \in p \mathbb{Z}_{p} /\left(p^{r_{2}}\right)$, so that $\bmod p$ the span $\left\langle\alpha^{1}, \alpha^{2}\right\rangle$ defines a line on the Pfaffian. With suitable local coordinate changes the congruences reduce to

$$
\begin{aligned}
& \overline{\mathrm{g}}\left(\begin{array}{cccc}
0 & a_{15} & 0 & 0 \\
0 & 0 & 0 & 0 \\
0 & 0 & 0 & 1 \\
0 & 0 & -1 & 0
\end{array}\right) \equiv 0 \quad\left(\bmod p^{r_{2}}\right) \\
& \overline{\mathrm{g}}\left(\begin{array}{cccc}
0 & a_{25} & a_{24} & 0 \\
0 & 0 & 0 & 1 \\
0 & 0 & 0 & 0 \\
0 & 0 & 0 & 0
\end{array}\right) \equiv 0 \quad\left(\bmod p^{r_{2}}\right) .
\end{aligned}
$$

Corollary 3.3. For a mixed lattice of type $\left(1, \ldots, 1, p^{r_{2}}, p^{r_{1}+r_{2}}\right)$ any flag $\left\langle\alpha^{1}\right\rangle \leq\left\langle\alpha^{1}, \alpha^{2}\right\rangle$ gives the same weight function.

Proof. As each of the lines and each of the points give the same weight function, so does any flag of any point-line combination. We can thus take the following vectors $\alpha^{1}=\left(a_{15}, a_{14}, a_{13}, a_{12}, a_{11}, 1\right)^{t}$ and $\alpha^{2}=\left(a_{25}, a_{24}, a_{23}, a_{22}, 1,0\right)^{t}$, where $a_{2 j} \in p \mathbb{Z}_{p} /\left(p^{r_{2}}\right), a_{1 j} \in p \mathbb{Z}_{p} /\left(p^{r_{1}+r_{2}}\right)$ for $2 \leq j \leq 5, a_{11} \in p \mathbb{Z}_{p} /\left(p^{r_{1}}\right)$. With suitable chance of local coordinates the congruence conditions look like

$$
\begin{gathered}
\overline{\mathrm{g}}\left(\begin{array}{cccc}
0 & a_{15} & 0 & 0 \\
-a_{15} & 0 & 0 & 0 \\
0 & 0 & 0 & 1 \\
0 & 0 & -1 & 0
\end{array}\right) \equiv 0 \quad\left(\bmod p^{r_{1}+r_{2}}\right) \\
\overline{\mathbf{g}}\left(\begin{array}{cccc}
0 & a_{25} & a_{24} & 0 \\
0 & 0 & 0 & 1 \\
0 & 0 & 0 & 0 \\
0 & 0 & 0 & 0
\end{array}\right) \equiv 0 \quad\left(\bmod p^{r_{2}}\right),
\end{gathered}
$$

from which we can read off the weight function to be $w^{\prime}\left(\Lambda^{\prime}\right)=6 r_{2}+5 r_{1}-$ $\min \left\{r_{1}, v_{p}\left(a_{15}\right)\right\}-\min \left\{r_{1}+r_{2}, v_{p}\left(a_{15}\right), v_{p}\left(a_{25}\right)+r_{1}, v_{p}\left(a_{24}\right)+r_{1}\right\}$.

However, with planes we need to be more careful. As planes are the highest dimensional linear subspace on the quadric, there are two families of planes, and as we shall see, these rulings do not behave equally; only one of them gives a different weight function. The variety of the planes is smooth, thus it is enough to consider a representative in each one of the rulings. 
Lemma 3.4. The plane generated by the vectors $\alpha^{1}=\left(1,0,0, a_{33}, a_{34}, a_{35}\right)^{t}$, $\alpha^{2}=\left(0,1,0, a_{23}, a_{24}, a_{25}\right)^{t}$ and $\alpha^{3}=\left(0,0,1, a_{13}, a_{14}, a_{15}\right)^{t}, a_{i j} \in p \mathbb{Z}_{p} /\left(p^{r_{3}}\right)$ lies on the Pfaffian $\bmod p$, but doesn't give a different weight function. For this half of the planes the weight function is $w^{\prime}\left(\Lambda^{\prime}\right)=7 r_{3}$ which is also the weight function for planes outside the Pfaffian. There are $\left(p^{2}+1\right)(p+1)$ such planes.

Proof. It is easy to see that the plane spanned by $\left\langle\alpha^{1}, \alpha^{2}, \alpha^{3}\right\rangle$ is on the Pfaffian $\bmod p$. The congruence conditions $\bmod p$ are $\overline{\mathbf{g}} \cdot M\left(\alpha^{i}\right) \equiv 0$ $\bmod p$ for $i=1,2,3$, and

$$
\begin{aligned}
& \mathbf{g}\left(\begin{array}{cccc}
0 & 1 & 0 & 0 \\
-1 & 0 & a_{33} & a_{34} \\
0 & -a_{33} & 0 & a_{35} \\
0 & -a_{34} & -a_{35} & 0
\end{array}\right) \equiv 0 \quad\left(\bmod p^{r_{3}}\right) \\
& \overline{\mathbf{g}}\left(\begin{array}{cccc}
0 & 0 & 1 & 0 \\
0 & 0 & a_{23} & a_{24} \\
-1 & -a_{23} & 0 & a_{25} \\
0 & -a_{24} & -a_{25} & 0
\end{array}\right) \equiv 0 \quad\left(\bmod p^{r_{3}}\right) \\
& \overline{\mathbf{g}}\left(\begin{array}{cccc}
0 & 0 & 0 & 1 \\
0 & 0 & a_{13} & a_{14} \\
0 & -a_{13} & 0 & a_{15} \\
-1 & -a_{14} & -a_{15} & 0
\end{array}\right) \equiv 0 \quad\left(\bmod p^{r_{3}}\right) .
\end{aligned}
$$

This set of equations has rank 4 , and thus the weight function is $w^{\prime}\left(\Lambda^{\prime}\right)=$ $7 r_{3}$ which is also the weight function for planes outside the Pfaffian.

Lemma 3.5. For the other $\left(p^{2}+1\right)(p+1)$ of planes the weight function is $w^{\prime}\left(\Lambda^{\prime}\right)=7 r_{3}-\min \left\{r_{3}, v_{p}\left(a_{15}\right), v_{p}\left(a_{25}\right), v_{p}\left(a_{24}\right), v_{p}\left(a_{35}\right), v_{p}\left(a_{34}\right), v_{p}\left(a_{33}\right)\right\}$.

Proof. The other family of planes can be represented by the span of the three vectors $\alpha^{1}=\left(a_{15}, a_{14}, a_{13}, 0,0,1\right)^{t}, \alpha^{2}=\left(a_{25}, a_{24}, a_{23}, 0,1,0\right)^{t}, \alpha^{3}=$ $\left(a_{35}, a_{34}, a_{33}, 1,0,0\right)$, where $a_{i j} \in p \mathbb{Z}_{p} /\left(p^{r_{3}}\right)$. With a suitable change of local coordinates the congruence conditions look like.

$$
\begin{aligned}
& \overline{\mathbf{g}}\left(\begin{array}{cccc}
0 & a_{15} & 0 & 0 \\
0 & 0 & 0 & 0 \\
0 & 0 & 0 & 1 \\
0 & 0 & -1 & 0
\end{array}\right) \equiv 0 \quad\left(\bmod p^{r_{3}}\right) \\
& \overline{\mathbf{g}}\left(\begin{array}{cccc}
0 & a_{25} & a_{24} & 0 \\
0 & 0 & 0 & 1 \\
0 & 0 & 0 & 0 \\
0 & 0 & 0 & 0
\end{array}\right) \equiv 0 \quad\left(\bmod p^{r_{3}}\right)
\end{aligned}
$$




$$
\overline{\mathrm{g}}\left(\begin{array}{cccc}
0 & a_{35} & a_{34} & a_{33} \\
0 & 0 & 0 & 0 \\
0 & 0 & 0 & 0 \\
0 & 0 & 0 & 0
\end{array}\right) \equiv 0 \quad\left(\bmod p^{r_{3}}\right)
$$

We can read off the weight function to be as claimed.

Corollary 3.6. For the mixed lattices containing the type $\left(1, \ldots, p^{r_{3}}, p^{r_{2}+r_{3}}, p^{r_{1}+r_{2}+r_{3}}\right) r_{3}>0$ and $r_{1}, r_{2} \geq 0$ we need to consider the planes that give a different weight function only. Other planes reduce this to the two-dimensional case.

Proof. For mixed lattices corresponding to the vectors $\alpha^{3}=$ $\left(1, a_{34}, a_{33}, a_{32}, a_{31}, a_{30}\right), \quad \alpha^{2}=\left(0,1, a_{23}, a_{22}, a_{21}, a_{20}\right), \quad \alpha^{1}=$ $\left(0,0,1, a_{12}, a_{11}, a_{10}\right)$ the rank of matrices on the level of planes is four.

Lemma 3.7. The weight function for mixed lattices where the plane is not one of the rank four planes is

$$
\begin{aligned}
& w^{\prime}\left(\Lambda^{\prime}\right)=7 r_{3}+6 r_{2}+5 r_{1}-\min \left\{r_{1}, v_{p}\left(a_{15}\right)\right\} \\
& -\min \left\{r_{1}+r_{2}+r_{3}, v_{p}\left(a_{15}\right), v_{p}\left(a_{25}\right)+r_{1}, v_{p}\left(a_{24}\right)+r_{1},\right. \\
& \left.\quad v_{p}\left(a_{35}\right)+r_{1}+r_{2}, v_{p}\left(a_{34}\right)+r_{1}+r_{2}, v_{p}\left(a_{33}\right)+r_{1}+r_{2}\right\} .
\end{aligned}
$$

Proof. We count over lattices of elementary divisor type $\left(1,1,1, p^{r_{3}}, p^{r_{2}+r_{3}}, p^{r_{1}+r_{2}+r_{3}}\right)$. The lattices $\Lambda^{\prime}$ lifting this flag are in one-to-one correspondence with the three of vectors encoded as columns of the matrix. Call the vectors $\alpha^{3}, \alpha^{2}$ and $\alpha^{1}$, respectively,

$$
\left(\begin{array}{ccc}
a_{35} & a_{25} & a_{15} \\
a_{34} & a_{24} & a_{14} \\
a_{33} & a_{23} & a_{13} \\
1 & a_{22} & a_{12} \\
0 & 1 & a_{11} \\
0 & 0 & 1
\end{array}\right)
$$

where $a_{35}, a_{34}, a_{33} \in p \mathbb{Z}_{p} /\left(p^{r_{3}}\right), a_{25}, a_{24}, a_{23} \in p \mathbb{Z}_{p} /\left(p^{r_{2}+r_{3}}\right), a_{22} \in$ $p \mathbb{Z}_{p} /\left(p^{r_{2}}\right), a_{15}, a_{14}, a_{13} \in p \mathbb{Z}_{p} /\left(p^{r_{1}+r_{2}+r_{3}}\right), a_{12} \in p \mathbb{Z}_{p} /\left(p^{r_{1}+r_{2}}\right), a_{11} \in p \mathbb{Z}_{p} /\left(p^{r_{1}}\right)$.

So what we are required to do is to solve the following congruences:

$$
\begin{array}{ll}
\overline{\mathbf{g}} \cdot M\left(\alpha^{3}\right) \equiv 0 & \left(\bmod p^{r_{3}}\right) \\
\overline{\mathbf{g}} \cdot M\left(\alpha^{2}\right) \equiv 0 & \left(\bmod p^{r_{2}+r_{3}}\right) \\
\overline{\mathbf{g}} \cdot M\left(\alpha^{1}\right) \equiv 0 & \left(\bmod p^{r_{1}+r_{2}+r_{3}}\right)
\end{array}
$$


simultaneously.

With suitable local coordinate changes the conditions reduce to

$$
\begin{gathered}
\mathbf{g}\left(\begin{array}{cccc}
0 & a_{15} & 0 & 0 \\
-a_{15} & 0 & 0 & 0 \\
0 & 0 & 0 & 1 \\
0 & 0 & -1 & 0
\end{array}\right) \equiv 0 \quad\left(\bmod p^{r_{1}+r_{2}+r_{3}}\right) \\
\mathbf{g}\left(\begin{array}{cccc}
0 & a_{25} & a_{24} & 0 \\
0 & 0 & 0 & 1 \\
0 & 0 & 0 & 0 \\
0 & 0 & 0 & 0
\end{array}\right) \equiv 0 \quad\left(\bmod p^{r_{2}+r_{3}}\right) \\
\mathbf{g}\left(\begin{array}{cccc}
0 & a_{35} & a_{34} & a_{33} \\
0 & 0 & 0 & 0 \\
0 & 0 & 0 & 0 \\
0 & 0 & 0 & 0
\end{array}\right) \equiv 0 \quad\left(\bmod p^{r_{3}}\right)
\end{gathered}
$$

The explicit weight function for this is

$$
\begin{aligned}
& w^{\prime}\left(\Lambda^{\prime}\right)=7 r_{3}+6 r_{2}+5 r_{1}-\min \left\{r_{1}, v_{p}\left(a_{15}\right)\right\} \\
& -\min \left\{r_{1}+r_{2}+r_{3}, v_{p}\left(a_{15}\right), v_{p}\left(a_{25}\right)+r_{1}, v_{p}\left(a_{24}\right)+r_{1},\right. \\
& \left.v_{p}\left(a_{35}\right)+r_{1}+r_{2}, v_{p}\left(a_{34}\right)+r_{1}+r_{2}, v_{p}\left(a_{33}\right)+r_{1}+r_{2}\right\} .
\end{aligned}
$$

Other types of mixed lattices containing $r_{3}$ are obtained by putting either of $r_{1}$ or $r_{2}$ equal to zero in the formula above.

\section{Generating functions}

Recall from (3) that the zeta function takes the shape

$$
\zeta_{F_{2,4}}^{\triangleleft}(s)=\zeta_{\mathbb{Z}^{4}, p}(s) \zeta_{p}(10 s-24) \cdot A\left(p, p^{-s}\right) .
$$

We can decompose the generating function $A\left(p, p^{-s}\right)$ further to run over lattices of fixed type, and write it as

$$
A\left(p, p^{-s}\right)=\sum_{I \subseteq\left\{1, \ldots, d^{\prime}-1\right\}} A_{I}\left(p, p^{-s}\right)
$$

where

$$
A_{I}\left(p, p^{-s}\right)=\sum_{\nu\left(\Lambda^{\prime}\right)=I} p^{d w\left(\Lambda^{\prime}\right)-s w^{\prime}\left(\Lambda^{\prime}\right)} \mu\left(\Lambda^{\prime}\right)
$$


$\Lambda^{\prime}$ is a representative lattice of type $I$, and $\mu\left(\Lambda^{\prime}\right)$ is its multiplicity.

However, the above is still not the optimal way to decompose things; we somehow need to involve the dependence on the geometric pieces which give a different weight function. A more subtle decomposition is needed.

Let us denote by $\left\{i^{*}\right\}, i=1,2,3$ those lattices of type $\{i\}$ that lift a given $i$-1-dimensional linear subspace of the projective space $\mathbb{P}^{5}$ that lies on the Pfaffian and accordingly by $A_{i^{*}}$ the generating function counting along these lattices. Similarly for mixed lattices. For instance, we have the lattices $\left\{1^{*}, 2^{*}\right\}$ which lift a flag of type $\mathcal{F}_{1,2}$ consisting of a line containing a point on the Pfaffian, and $A_{1^{*}, 2^{*}}$ for the corresponding generating function. In short, $A_{I}$ denotes the generating function along lattices of type $I$ and the extra stars on elements $I$ just tell if this part of the lattice has lifted a flag variety that lies on the Pfaffian hypersurface.

\subsection{Indexing}

The indexing in this case has to be done carefully in order to take all the possibilities into account and we shall use the indexing set $I \subset$ $\left\{1,2,3,4,5,1^{*}, 2^{*}, 3^{*}\right\}$, such that if $i \in I$ then $i^{*} \notin I$ and if $j^{*} \in I$ then $k \notin I$ when $k \leq j$.

Now the generating function $A\left(p, p^{-s}\right)$ can be split into parts

$$
\begin{aligned}
A\left(p, p^{-s}\right) & =\sum_{I \subseteq\{1, \ldots, 5\}} c_{I, p} A_{I}\left(p, p^{-s}\right)+\sum_{\substack{I=1^{*} \cup J_{2} \\
J_{2} \subseteq\{2, \ldots, 5\}}} c_{I, p} A_{I}\left(p, p^{-s}\right)+\sum_{\substack{I=J_{1} \cup 2^{*} \cup J_{2} \\
J_{1} \subseteq\left\{1^{*}\right\} \\
J_{2} \subseteq\{3,4,5\}}} c_{I, p} A_{I}\left(p, p^{-s}\right) \\
& +\sum_{\substack{I=J_{1} \cup 3^{*} \cup J_{2} \\
J_{1} \subseteq\left\{1^{*}, 2^{*}\right\} \\
J_{2} \subseteq\{4,5\}}} c_{I, p} A_{I}\left(p, p^{-s}\right)
\end{aligned}
$$

where $A_{I}\left(p, p^{-s}\right)$ are as in (13) and $c_{I, p}$ are coefficients depending on the type of lattice and number of $\mathbb{F}_{p}$-points on certain varieties, and can be explicitly written down as polynomials in $p$.

In order to write down the coefficients $c_{I, p}$ we need some definitions. Let $\mathfrak{n}_{i}(p)$ be the number of $\mathbb{F}_{p}$-rational points of the Fano varieties of $(i-1)$ dimensional subspaces on the Pfaffian hypersurface. In the case of planes, however, we need to take the number of planes on the ruling that gave the different weight function. Explicitly

$$
\begin{aligned}
& \mathfrak{n}_{1}(p)=\left(p^{2}+1\right)\left(p^{2}+p+1\right), \\
& \mathfrak{n}_{2}(p)=(p+1)\left(p^{2}+1\right)\left(p^{2}+p+1\right), \\
& \mathfrak{n}_{3}(p)=\left(p^{2}+1\right)(p+1) .
\end{aligned}
$$


So in this example we have $\mathfrak{n}_{3}(p)=\left(p^{2}+1\right)(p+1)$ but really there are $\mathfrak{P}_{3}(p)=2\left(p^{2}+1\right)(p+1) \mathbb{F}_{p}$-points on the Fano variety of planes on a quadric four-fold.

Now we can explicitly describe the coefficients $c_{I, p}$ appearing in the generating function. Let us denote by $b_{I}(p)$ the number of points on the flag variety defined by lattices of type $I$. We also write $I-k$ to mean the type of lattice we get if we subtract from each index $i \in I$, the number $k$, so if $I=\{4,5,6\}$ then $I-3=\{1,2,3\}$. With this notation we have

1) If $I \subseteq\{1, \ldots, 5\}$ and $I=\left\{i_{1}, \ldots, i_{n}\right\}$, then

$$
\begin{aligned}
c_{I, p} & =\left(\begin{array}{c}
6-i_{1}-\cdots-i_{n-1} \\
i_{n-1}-i_{n}
\end{array}\right)_{p} \ldots\left(\begin{array}{c}
6-i_{1} \\
i_{1}-i_{2}
\end{array}\right)_{p}\left(\left(\begin{array}{c}
6 \\
i_{1}
\end{array}\right)_{p}-\mathfrak{n}_{i_{1}}(p)\right) \\
& =b_{I}(p)-b_{I-i_{1}}(p) \mathfrak{n}_{i_{1}}(p) .
\end{aligned}
$$

2) If $I=\left\{i_{1}, \ldots, i_{n}, k^{*}, j_{1}, \ldots, j_{r}\right\}$, then

$$
\begin{aligned}
c_{I, p} & =\left(\begin{array}{c}
6-k-\cdots-j_{r-1} \\
j_{r}-j_{r-1}
\end{array}\right)_{p} \ldots\left(\begin{array}{c}
6-k-j_{1} \\
j_{2}-j_{1}
\end{array}\right)_{p} \\
& \cdot\left(\left(\begin{array}{c}
6-k \\
k-j_{1}
\end{array}\right)_{p} \mathfrak{n}_{k}(p)\left(\begin{array}{c}
k-i_{1} \cdots-i_{n} \\
i_{n}-i_{n-1}
\end{array}\right)_{p} \ldots\left(\begin{array}{c}
k \\
i_{1}
\end{array}\right)_{p}-\mathfrak{n}_{j_{1}}(p)\left(\begin{array}{c}
j_{1}-i_{1}-\ldots i_{n} \\
k-i_{n}
\end{array}\right)_{p} \ldots\left(\begin{array}{c}
j_{1} \\
i_{1}
\end{array}\right)_{p}\right) \\
& =b_{J_{2}-k}(p) \mathfrak{n}_{k}(p) b_{J_{1}}(p)-b_{J_{2}-\left(k-j_{1}\right)}(p) \mathfrak{n}_{k+1}(p) b_{J_{1} \cup k}(p) .
\end{aligned}
$$

To see exactly where in the zeta function we have the dependence on the $\mathbb{F}_{p}$-points of a Fano variety, let us now rearrange (14) into the form

$A\left(p, p^{-s}\right)=W_{0}\left(p, p^{-s}\right)+\mathfrak{n}_{1}(p) W_{1}\left(p, p^{-s}\right)+\mathfrak{n}_{2}(p) W_{2}\left(p, p^{-s}\right)+\mathfrak{n}_{3}(p) W_{3}\left(p, p^{-s}\right)$.

From the formulae for the coefficients $c_{I, p}$ we see that the only $c_{I, p}$ that don't depend on any of the $\mathfrak{n}_{i}(p)$ come from the first summand, and so we get

$$
W_{0}\left(p, p^{-s}\right)=\sum_{I \subseteq\{1, \ldots, 5\}} b_{I}(p) A_{I}\left(p, p^{-s}\right) .
$$

We also observe that $\mathfrak{n}_{1}(p)$ appears only in the first two summands in (14) and thus 


$$
\begin{aligned}
& W_{1}\left(p, p^{-s}\right)=\sum_{\substack{I=1^{*} \cup J_{2} \\
J_{2} \subseteq\{2, \ldots, 5\}}} b_{I-1}(p) A_{I}\left(p, p^{-s}\right)-\sum_{\substack{I=1 \cup J_{2} \\
J_{2} \subseteq\{2, \ldots, 5\}}} b_{I-1}(p) A_{I}\left(p, p^{-s}\right) \\
& =\sum_{I \subseteq\{2, \ldots, 5\}} b_{I-1}(p)\left(A_{1^{*} \cup I}\left(p, p^{-s}\right)-A_{1 \cup I}\left(p, p^{-s}\right)\right) \text {. }
\end{aligned}
$$

Similarly we extract the dependence on $\mathfrak{n}_{2}(p)$ and notice that it appears only in the first two summands of (14)

$$
\begin{aligned}
W_{2}\left(p, p^{-s}\right) & =\sum_{\substack{I=J_{1} \cup 2^{*} \cup J_{2} \\
J_{1} \subseteq\left\{1^{*}\right\} \\
J_{2} \subseteq\{3,4,5\}}} b_{J_{2}-2}(p) b_{J_{1}}(p) A_{I}\left(p, p^{-s}\right)-\sum_{\substack{I=J_{1} \cup 2 \cup J_{2} \\
J_{1} \subseteq\left\{1^{*}\right\} \\
J_{2} \subseteq\{3,4,5\}}} b_{J_{2}-2}(p) b_{J_{1}}(p) A_{I}\left(p, p^{-s}\right) \\
& =\sum_{\substack{J_{1} \subseteq\left\{1^{*}\right\} \\
J_{2} \subseteq\{3,4,5\}}} b_{J_{2}-2}(p) b_{J_{1}}(p)\left(A_{J_{1} \cup 2^{*} \cup J_{2}}\left(p, p^{-s}\right)-A_{J_{1} \cup 2 \cup J_{2}}\left(p, p^{-s}\right)\right)
\end{aligned}
$$

And finally we do the same for $\mathfrak{n}_{3}(p)$ and get

$$
\begin{aligned}
W_{3}\left(p, p^{-s}\right) & =\sum_{\substack{I=J_{1} \cup 3^{*} \cup J_{2} \\
J_{1} \subseteq\left\{1^{*}, 2^{*}\right\} \\
J_{2} \subseteq\{4,5\}}} b_{J_{2}-3}(p) b_{J_{1}}(p) A_{I}\left(p, p^{-s}\right)-\sum_{\substack{I=J_{1} \cup 3 \cup J_{2} \\
J_{1} \subseteq\left\{1^{*}, 2^{*}\right\} \\
J_{2} \subseteq\{4,5\}}} b_{J_{2}-3}(p) b_{J_{1}}(p) A_{I}\left(p, p^{-s}\right) \\
& =\sum_{\substack{J_{1} \subseteq\left\{1^{*}, 2^{*}\right\} \\
J_{2} \subseteq\{4,5\}}} b_{J_{2}-3}(p) b_{J_{1}}(p)\left(A_{J_{1} \cup 3^{*} \cup J_{2}}\left(p, p^{-s}\right)-A_{J_{1} \cup 3 \cup J_{2}}\left(p, p^{-s}\right)\right)
\end{aligned}
$$

\subsection{Igusa factors}

We observe the factor $I_{n}(\mathbf{U})$ appearing frequently in the formulae. In this section we see that it is a natural part of the zeta functions.

Definition 4.1. In an expression of the form

$$
W_{i}(X, Y)=I_{d^{\prime}-i-1}\left(X_{d^{\prime}-1}, \ldots, X_{i+1}\right) E_{i}\left(X_{i}, Y_{i}\right) I_{i-1}\left(Y_{i-1}, \ldots, Y_{1}\right)
$$

we shall call the factor $I_{d^{\prime}-i-1}(\mathbf{X})$ the upper Igusa factor and the factor $I_{i-1}(\mathbf{Y})$ the lower Igusa factor.

Some of Voll's work has concerned the Igusa factor, for instance his formula for the normal zeta function of the so-called Grenham groups in [13] is completely of this form. There he also observed the existence of the upper 
Igusa factor in the formula of $W_{1}\left(p, p^{-s}\right)$. The existence of the lower Igusa factor is recorded here publicly for the first time. However, it did appear in the calculation of the Segre example [11] where the Pfaffian hypersurface was the Segre surface.

Let us now start to calculate and determine where these factors come from.

For completeness we shall calculate $W_{0}\left(p, p^{-s}\right)$ here again, and in this context using the $\mu$-function.

Lemma 4.2 (Voll).

$$
W_{0}\left(p, p^{-s}\right)=\sum_{I \subseteq\{1, \ldots, 5\}} b_{I}(p) A_{I}\left(p, p^{-s}\right)=\sum_{I \subseteq\{1, \ldots, 5\}} b_{I}\left(p^{-1}\right) \prod_{i \in I} \frac{X_{i}}{1-X_{i}},
$$

where $X_{i}=p^{i(10-i)-(4+i) s}$ for $i=1, \ldots, 5$.

Proof. Using the lemma 2.7 we can write

$$
\begin{aligned}
& \sum_{I \subseteq\{1, \ldots, 5\}} b_{I}(p) A_{I}\left(p, p^{-s}\right) \\
= & \sum_{I \subseteq\{1, \ldots, 5\}} b_{I}(p) \prod_{i \in I} \sum_{r_{i}=1}^{\infty} p^{4 i r_{i}-(4+i) r_{i} s} p^{-\operatorname{dim} \mathcal{F}_{I}} p^{\sum_{i \in I}(6-i) i r_{i}} \\
= & \sum_{I \subseteq\{1, \ldots, 5\}} b_{I}\left(p^{-1}\right) \prod_{i \in I} \sum_{r_{i}=1}^{\infty} p^{i(10-i) r_{i}-(4+i) r_{i} s} \\
= & \sum_{I \subseteq\{1, \ldots, 5\}} b_{I}\left(p^{-1}\right) \prod_{i \in I} \frac{p^{i(10-i)-(4+i) s}}{1-p^{i(10-i)-(4+i) s}} .
\end{aligned}
$$

\subsection{Extracting the upper Igusa factor}

For simplicity of notation we shall ignore the variables $p$ and $p^{-s}$ from the generating functions and use only $A_{I}$ to denote $A_{I}\left(p, p^{-s}\right)$. We shall also from now on forget the $p$-adic valuations $v_{p}\left(a_{i j}\right)$ in the min-expressions and will write, for example, $\min \left\{r_{1}, a_{15}\right\}$ instead of $\min \left\{r_{1}, v_{p}\left(a_{15}\right)\right\}$.

We can first simplify our formulae by summing out most of the variables. We do this in the next two lemmas.

\section{Lemma 4.3.}

$$
\left(A_{J_{1} \cup i^{*} \cup J_{2}}-A_{J_{1} \cup i \cup J_{2}}\right)=p^{-\operatorname{dim} \mathcal{F}_{J_{2}-i}} \tilde{A}_{J_{2}} \cdot\left(A_{J_{1} \cup i^{*}}-A_{J_{1} \cup i}\right),
$$

where $\tilde{A}_{J_{2}}=\prod_{j_{2} \in J_{2}} \frac{X_{j_{2}}}{1-X_{j_{2}}}$ and $X_{j_{2}}=p^{\left(10-j_{2}\right) j_{2}-s\left(4+j_{2}\right)}$ as before. 
Proof.

$$
\begin{aligned}
& \left(A_{J_{1} \cup i^{*} \cup J_{2}}-A_{J_{1} \cup i \cup J_{2}}\right)=\prod_{j_{2} \in J_{2}} \sum_{r_{j_{2}}=1}^{\infty} p^{4 j_{2} r_{j_{2}}-s\left(4+j_{2}\right) r_{j_{2}}} \sum_{r_{i}=1}^{\infty} p^{4 i r_{i}-s(4+i) r_{i}} . \\
& \cdot \prod_{j_{1} \in J_{1}} \sum_{r_{j_{1}}=1}^{\infty} p^{4 j_{1} r_{j_{1}}-s\left(4+j_{1}\right) r_{j_{1}}} \prod_{\substack{i, j \in J_{1} \cup i \cup J_{2} \\
j \leq i}} \sum_{a_{i j}=1}^{\sum_{k=i}^{j} r_{k}} \mu\left(\sum_{k=i}^{j} r_{k}, a_{i j}\right) . \\
& \cdot\left(p^{s \min \left\{\sum_{j_{1} \in J_{1}} r_{j_{1}}+r_{i}, a_{i j}+r_{1}+\cdots+r_{i-1}\right\}}-p^{s \min \left\{\sum_{j_{1} \in J_{1}} r_{j_{1}}, a_{i j}+r_{1}+\cdots+r_{i-2}\right\}}\right) .
\end{aligned}
$$

We can now sum up all those $\mu$-functions that depend only on $r_{j_{2}}$ and from those we get $p^{-\operatorname{dim} \mathcal{F}_{J_{2}-i}} p^{\sum_{j_{2} \in J_{2}}\left(6-j_{2}-i\right) j_{2} r_{j_{2}}}$. Moreover we can use the property of $\mu$ that

$$
\sum_{a_{i j}=1}^{r_{i}+\cdots+r_{j}} \mu\left(r_{i}+\cdots+r_{j}, a_{i j}\right)=p^{r_{k}+\cdots+r_{j}} \sum_{a_{i j}=1}^{r_{i}+\cdots+r_{k-1}} \mu\left(r_{i}+\cdots+r_{k-1}, a_{i j}\right)
$$

to extract the $r_{j_{2}}$ from the $\mu$-part as well, as these don't appear in the minexpressions. Then we get $p^{\sum_{j_{2} \in J_{2}} i j_{2} r_{j_{2}}}$ out of the $\mu$ 's.

In conclusion, we have extracted

$$
p^{-\operatorname{dim} \mathcal{F}_{J_{2}-i}} p^{\sum_{j_{2} \in J_{2}}\left(6-j_{2}\right) j_{2} r_{j_{2}}},
$$

and we can rewrite

$$
\begin{aligned}
\left(A_{J_{1} \cup i^{*} \cup J_{2}}-A_{J_{1} \cup i \cup J_{2}}\right) & =\prod_{j_{2} \in J_{2}} \sum_{r_{j_{2}}=1}^{\infty} p^{4 j_{2} r_{j_{2}}-s\left(4+j_{2}\right) r_{j_{2}}} p^{-\operatorname{dim} \mathcal{F}_{J_{2}-i}} p^{\sum_{j_{2} \in J_{2}}\left(6-j_{2}\right) j_{2} r_{j_{2}}}\left(A_{J_{1} \cup i^{*}}-A_{J_{1} \cup i}\right) \\
& =p^{-\operatorname{dim} \mathcal{F}_{J_{2}-i}} \prod_{j_{2} \in J_{2}} \sum_{r_{j_{2}}=1}^{\infty} p^{\left(10-j_{2}\right) r_{j_{2}}-s\left(4+j_{2}\right) r_{j_{2}}}\left(A_{J_{1} \cup i^{*}}-A_{J_{1} \cup i}\right) \\
& =p^{-\operatorname{dim} \mathcal{F}_{J_{2}-i}} \tilde{A}_{J_{2}} \cdot\left(A_{J_{1} \cup i^{*}}-A_{J_{1} \cup i}\right)
\end{aligned}
$$

\section{Lemma 4.4.}

$$
\begin{aligned}
W_{i} & =\sum_{\substack{J_{1} \subseteq\left\{1^{*}, \ldots, i-1^{*}\right\} \\
J_{2} \subseteq\{i+1, \ldots, 5\}}} b_{J_{2}-i}(p) b_{J_{1}}(p)\left(A_{J_{1} \cup i^{*} \cup J_{2}}-A_{J_{1} \cup i \cup J_{2}}\right) \\
& =I_{5-i}\left(X_{i+1}, \ldots, X_{5}\right) \cdot \sum_{J_{1} \subseteq\left\{1^{*}, \ldots, i-1^{*}\right\}} b_{J_{1}}(p)\left(A_{J_{1} \cup i^{*}}-A_{J_{1} \cup i}\right)
\end{aligned}
$$

with the poles $X_{k}=p^{k(10-k)-(4+k) s}$ for $k=i+1, \ldots, 5$. 
Proof.

$$
\begin{aligned}
W_{i} & =\sum_{\substack{J_{1} \subseteq\left\{1^{*}, \ldots, i-1^{*}\right\} \\
J_{2} \subseteq\{i+1, \ldots, 5\}}} b_{J_{2}-i}(p) b_{J_{1}}(p)\left(A_{J_{1} \cup i^{*} \cup J_{2}}-A_{J_{1} \cup i \cup J_{2}}\right) \\
& =\sum_{\substack{J_{1} \subseteq\left\{1^{*}, \ldots, i-1^{*}\right\} \\
J_{2} \subseteq\{i+1, \ldots, 5\}}} b_{J_{2}-i}(p) b_{J_{1}}(p) p^{-\operatorname{dim} \mathcal{F}_{J_{2}-i}} A_{J_{2}} \cdot\left(A_{J_{1} \cup i^{*}}-A_{J_{1} \cup i}\right) \\
& =\sum_{J_{2} \subseteq\{i+1, \ldots, 5\}} \sum_{J_{1} \subseteq\left\{1^{*}, \ldots, i-1^{*}\right\}} b_{J_{2}-i}\left(p^{-1}\right) b_{J_{1}}(p) A_{J_{2}} \cdot\left(A_{J_{1} \cup i^{*}}-A_{J_{1} \cup i}\right) \\
& =\sum_{J_{2} \subseteq\{i+1, \ldots, 5\}} b_{J_{2}-i}\left(p^{-1}\right) A_{J_{2}} \sum_{J_{1} \subseteq\left\{1^{*}, \ldots, i-1^{*}\right\}} b_{J_{1}}(p)\left(A_{J_{1} \cup i^{*}}-A_{J_{1} \cup i}\right) \\
& =I_{5-i}\left(X_{i+1}, \ldots, X_{5}\right) \cdot \sum_{J_{1} \subseteq\left\{1^{*}, \ldots, i-1^{*}\right\}} b_{J_{1}}(p)\left(A_{J_{1} \cup i^{*}}-A_{J_{1} \cup i}\right) .
\end{aligned}
$$

Corollary 4.5. Using the above lemma we can write the rational functions

$$
\begin{aligned}
& W_{0}\left(p, p^{-s}\right)=\sum_{I \subseteq\{1, \ldots, 5\}} b_{I}\left(p^{-1}\right) \prod_{i \in I} \frac{X_{i}}{1-X_{i}}=I_{5}\left(X_{1}, \ldots, X_{5}\right) . \\
& W_{1}\left(p, p^{-s}\right)=I_{4}\left(X_{2}, \ldots, X_{5}\right) \cdot\left(A_{1^{*}}-A_{1}\right) \\
& W_{2}\left(p, p^{-s}\right)=I_{3}\left(X_{3}, X_{4}, X_{5}\right) \cdot \sum_{J_{1} \subseteq\left\{1^{*}\right\}} b_{J_{1}}(p)\left(A_{J_{1} \cup 2^{*}}-A_{J_{1} \cup 2}\right) \\
& W_{3}\left(p, p^{-s}\right)=I_{2}\left(X_{4}, X_{5}\right) \cdot \sum_{J_{1} \subseteq\left\{1^{*}, 2^{*}\right\}} b_{J_{1}}(p)\left(A_{J_{1} \cup 3^{*}}-A_{J_{1} \cup 3}\right),
\end{aligned}
$$

where $X_{i}=p^{(10-i) i-(4+i) s}$ for $i=1, \ldots, 5$.

Thus it is enough to consider the sums

$$
\sum_{J_{1} \subseteq\left\{1^{*}, \ldots, i-1^{*}\right\}} b_{J_{1}}(p)\left(A_{J_{1} \cup i^{*}}-A_{J_{1} \cup i}\right),
$$

and that is what we shall do in the next section.

\subsection{The lower Igusa factor}

From the corollary 4.5 we recall the form of $W_{2}\left(p, p^{-s}\right)$ as

$$
W_{2}\left(p, p^{-s}\right)=I_{3}\left(X_{3}, X_{4}, X_{5}\right) \cdot \sum_{J_{1} \subseteq\left\{1^{*}\right\}} b_{J_{1}}(p)\left(A_{J_{1} \cup 2^{*}}-A_{J_{1} \cup 2}\right)
$$


Here we need to calculate two terms, namely $\left(A_{2^{*}}-A_{2}\right)$ and $\left(A_{1^{*}, 2^{*}}-\right.$ $\left.A_{1^{*}, 2}\right)$.

We shall start with the latter. This example demonstrates very well the general idea and isn't too hard to do explicitly.

Lemma 4.6.

$$
\left(A_{1^{*}, 2^{*}}-A_{1^{*}, 2}\right)=p^{-\operatorname{dim} \mathcal{F}_{\{1\}}} \frac{Y_{1}}{1-Y_{1}}\left(A_{2^{*}}-A_{2}\right),
$$

where $Y_{1}=p^{8-3 s}$.

Corollary 4.7.

$$
\sum_{J_{1} \subseteq\left\{1^{*}\right\}} b_{J_{1}}(p)\left(A_{J_{1} \cup 2^{*}}-A_{J_{1} \cup 2}\right)=I_{1}\left(Y_{1}\right)\left(A_{2^{*}}-A_{2}\right) .
$$

Proof. (Proof of corollary 4.7)

$$
\begin{aligned}
\sum_{J_{1} \subseteq\left\{1^{*}\right\}} b_{J_{1}}(p)\left(A_{J_{1} \cup 2^{*}}-A_{J_{1} \cup 2}\right) & =\left(1+b_{1}(p) p^{-1} \frac{Y_{1}}{1-Y_{1}}\right)\left(A_{2^{*}}-A_{2}\right) \\
& =I_{1}\left(Y_{1}\right)\left(A_{2^{*}}-A_{2}\right)
\end{aligned}
$$

by definition of the Igusa factor.

So we have proved that $W_{2}\left(p, p^{-s}\right)$ is of the form claimed. It is left to prove the lemma. This is the first example of a process I call extracting the lower Igusa factor.

Proof. (Proof of lemma 4.6) For simplicity, let us write $T:=p^{-s}$.

$$
\begin{aligned}
A_{1^{*}, 2^{*}}-A_{1^{*}, 2}= & \sum_{r_{2}=1}^{\infty} p^{8 r_{2}} T^{6 r_{2}} \sum_{r_{1}=1}^{\infty} p^{4 r_{1}} T^{5 r_{1}} \sum_{a_{15}, a_{14}, a_{13}, a_{12}=1}^{r_{1}+r_{2}} \mu\left(r_{1}+r_{2} ; a_{15}, a_{14}, a_{13}, a_{12}\right) \\
& \sum_{a_{11}=1}^{r_{1}} \mu\left(r_{1}, a_{11}\right) \sum_{a_{25}, a_{24}, a_{23}, a_{22}=1}^{r_{2}} \mu\left(r_{2} ; a_{25}, a_{24}, a_{23}, a_{22}\right) . \\
& \cdot\left(T^{-\min \left\{r_{1}, a_{15}\right\}-\min \left\{r_{1}+r_{2}, a_{15}, a_{25}+r_{1}, a_{24}+r_{1}\right\}}-T^{-2 \min \left\{r_{1}, a_{15}\right\}}\right) .
\end{aligned}
$$

First we can see that some variables are independent and can be summed separately. We shall write $\sum_{a_{11}=1}^{r_{1}} \mu\left(r_{1}, a_{11}\right)=p^{r_{1}-1}$ and more importantly

$\sum_{a_{15}, a_{14}, a_{13}, a_{12}=1}^{r_{1}+r_{2}} \mu\left(r_{1}+r_{2} ; a_{15}, a_{14}, a_{13}, a_{12}\right)=p^{3 r_{1}} \sum_{a_{14}, a_{13}, a_{12}=1}^{r_{2}} \mu\left(r_{2} ; a_{14}, a_{13}, a_{12}\right) \sum_{a_{15}=1}^{r_{1}+r_{2}} \mu\left(r_{1}+r_{2}, a_{15}\right)$. 
Denoting $\mathbf{a}=\left(a_{14}, a_{13}, a_{12}, a_{25}, a_{24}, a_{23}, a_{22}\right)$ and inserting the above two simplifications we can write our summations as

$$
\begin{aligned}
A_{1^{*}, 2^{*}}-A_{1^{*}, 2}= & p^{-1} \sum_{r_{2}=1}^{\infty} p^{4 r_{2}} T^{6 r_{2}} \sum_{\mathbf{a}=1}^{r_{2}} \mu\left(r_{2} ; \mathbf{a}\right) \sum_{r_{1}=1}^{\infty} p^{8 r_{1}} T^{5 r_{1}} \sum_{a_{15}=1}^{r_{1}+r_{2}} \mu\left(r_{1}+r_{2}, a_{15}\right) . \\
& \cdot\left(T^{-\min \left\{r_{1}, a_{15}\right\}-\min \left\{r_{1}+r_{2}, a_{15}, a_{25}+r_{1}, a_{24}+r_{1}\right\}}-T^{-2 \min \left\{r_{1}, a_{15}\right\}}\right) .
\end{aligned}
$$

Write

$$
\begin{aligned}
B\left(r_{2}\right) & =\sum_{r_{1}=1}^{\infty} p^{8 r_{1}} T^{5 r_{1}} \sum_{\substack{a_{15}=1 \\
r_{1}+r_{2}}} \mu\left(r_{1}+r_{2}, a_{15}\right) . \\
& \cdot\left(T^{-\min \left\{r_{1}, a_{15}\right\}-\min \left\{r_{1}+r_{2}, a_{15}, a_{25}+r_{1}, a_{24}+r_{1}\right\}}-T^{-2 \min \left\{r_{1}, a_{15}\right\}}\right)
\end{aligned}
$$

and start to decompose this according $r_{1}=1$ and $r_{1} \geq 2$, in the latter case change the variable $r_{1}^{\prime}=1+r_{1}$ :

$$
\begin{aligned}
B\left(r_{2}\right) & =p^{8} T^{5} \sum_{a_{15}=1}^{1+r_{2}} \mu\left(1+r_{2}, a_{15}\right) \cdot \\
& \cdot\left(T^{-1-\min \left\{1+r_{2}, a_{15}, a_{25}+1, a_{24}+1\right\}}-T^{-2 \min \left\{1, a_{15}\right\}}\right) \\
& +p^{8} T^{5} \sum_{r_{1}^{\prime}=1}^{\infty} p^{8 r_{1}} T^{5 r_{1}} \sum_{a_{15}=1}^{1+r_{1}^{\prime}+r_{2}} \mu\left(1+r_{1}^{\prime}+r_{2}, a_{15}\right) . \\
& \cdot\left(T^{-\min \left\{1+r_{1}^{\prime}, a_{15}\right\}-\min \left\{1+r_{1}^{\prime}+r_{2}, a_{15}, a_{25}+r_{1}^{\prime}+1, a_{24}+r_{1}^{\prime}+1\right\}}-T^{-2 \min \left\{1+r_{1}^{\prime}, a_{15}\right\}}\right) .
\end{aligned}
$$

Now if $a_{15}=1$ all the min-expressions will take value 1 and so the subtraction will cancel all these parts. Thus we can assume that $a_{15} \geq 2$. Moreover, we can change the range of summation

$$
\sum_{a_{15}=2}^{1+r_{1}^{\prime}} \mu\left(1+r_{1}^{\prime}, a_{15}\right)=\sum_{a_{15}=1}^{r_{1}^{\prime}} \mu\left(r_{1}^{\prime}, a_{15}\right)
$$

and note that $\min \left\{1+r_{1}^{\prime}, 1+a_{15}\right\}=1+\min \left\{r_{1}^{\prime}, a_{15}\right\}$. Now we can write the summations in the form

$$
\begin{aligned}
B\left(r_{2}\right) & =p^{8} T^{3} \sum_{a_{15}=1}^{r_{2}} \mu\left(r_{2}, a_{15}\right) \cdot \\
& \cdot\left(T^{-\min \left\{r_{2}, a_{15}, a_{25}, a_{24}\right\}}-1\right) \\
& +p^{8} T^{3} \sum_{r_{1}^{\prime}=1}^{\infty} p^{8 r_{1}} T^{5 r_{1}} \sum_{a_{15}=1}^{r_{1}^{\prime}+r_{2}} \mu\left(r_{1}^{\prime}+r_{2}, a_{15}\right) . \\
& \cdot\left(T^{-\min \left\{r_{1}^{\prime}, a_{15}\right\}-\min \left\{r_{1}^{\prime}+r_{2}, a_{15}, a_{25}+r_{1}^{\prime}, a_{24}+r_{1}^{\prime}\right\}}-T^{-2 \min \left\{r_{1}^{\prime}, a_{15}\right\}}\right) .
\end{aligned}
$$


Inserting this back to $A_{1^{*}, 2^{*}}-A_{1^{*}, 2}$ we obtain that

$$
\left(1-p^{8} T^{3}\right)\left(A_{1^{*}, 2^{*}}-A_{1^{*}, 2}\right)=p^{-1} p^{8} T^{3}\left(A_{2^{*}}-A_{2}\right),
$$

and thus

$$
\left(A_{1^{*}, 2^{*}}-A_{1^{*}, 2}\right)=p^{-1} \frac{Y_{1}}{1-Y_{1}}\left(A_{2^{*}}-A_{2}\right)
$$

where $Y_{1}=p^{8} T^{3}$ as claimed.

Calculating $W_{3}\left(p, p^{-s}\right)$ is very similar to $W_{2}\left(p, p^{-s}\right)$. We just have some more summands to consider.

\section{Proposition 4.8 .}

$$
\sum_{J_{1} \subseteq\left\{1^{*}, 2^{*}\right\}} b_{J_{1}}(p)\left(A_{J_{1} \cup 3^{*}}-A_{J_{1} \cup 3}\right)=I_{2}\left(Y_{1}, Y_{2}\right) \cdot\left(A_{3^{*}}-A_{3}\right) .
$$

Let us state the lemmas needed

\section{Lemma 4.9.}

$$
\left(A_{1^{*} \cup 3^{*}}-A_{1^{*} \cup 3}\right)=p^{-\operatorname{dim} \mathcal{F}_{1}} \frac{Y_{1}}{1-Y_{1}}\left(A_{3^{*}}-A_{3}\right)
$$

\section{Lemma 4.10.}

$$
\left(A_{2^{*} \cup 3^{*}}-A_{2^{*} \cup 3}\right)=p^{-\operatorname{dim} \mathcal{F}_{2}} \frac{Y_{2}}{1-Y_{2}}\left(A_{3^{*}}-A_{3}\right)
$$

\section{Lemma 4.11.}

$$
\left(A_{1^{*}, 2^{*} \cup 3^{*}}-A_{1^{*}, 2^{*} \cup 3}\right)=p^{-\operatorname{dim} \mathcal{F}_{1,2}} \frac{Y_{2}}{1-Y_{2}} \frac{Y_{1}}{1-Y_{1}}\left(A_{3^{*}}-A_{3}\right)
$$

Proof. The above three lemmas are proved in exactly the same way as lemma 4.6 - in the proof of lemma 4.11 we just need to do the procedure twice.

Proof. (of proposition 4.8) By the above lemmas

$$
\begin{aligned}
\sum_{J_{1} \subseteq\left\{1^{*}, 2^{*}\right\}} b_{J_{1}}(p)\left(A_{J_{1} \cup 3^{*}}-A_{J_{1} \cup 3}\right) & =\sum_{J_{1} \subseteq\left\{1^{*}, 2^{*}\right\}} b_{J_{1}}\left(p^{-1}\right) \prod_{j_{1} \in J_{1}} \frac{Y_{j_{1}}}{1-Y_{j_{1}}} \\
& =I_{2}\left(Y_{1}, Y_{2}\right) \cdot\left(A_{3^{*}}-A_{3}\right) .
\end{aligned}
$$




\subsection{The exceptional factor and final formulae}

Finally we need to calculate the exceptional factor $E_{i}=\left(A_{i^{*}}-A_{i}\right)$.

Proposition 4.12. Let $d=h\left(G^{a b}\right)$ be the torsion-free rank of the abelianisation and $d^{\prime}=h(Z(G))$ the torsion-free rank of the centre. Let $n_{i}=c_{i}+d_{i}$ where $n_{i}$ is the dimension of the space of $i$-1-dimensional linear subspace in $\mathbb{P}^{d^{\prime}-1}, d_{i}$ the dimension of the Fano variety of $i-1$-dimensional linear subspace of the Pfaffian, and $c_{i}$ the codimension of the same object. Then

$$
\begin{aligned}
A_{i^{*}}-A_{i} & =\sum_{r_{i}=1}^{\infty} \sum_{b_{1}=1}^{r_{i}} \sum_{b_{2}=1}^{r_{i}} \cdots \sum_{b_{n_{i}}=1}^{r_{i}} \mu\left(r_{i} ; b_{1}, b_{2}, \ldots, b_{n_{i}}\right) p^{i d r_{i}} T^{(d+i) r_{i}}\left(T^{-t \min \left\{r_{i}, b_{1}, b_{2}, \ldots, b_{c_{i}}\right\}}-1\right) \\
& =\frac{p^{d i} T^{d+i-t}\left(1-T^{t}\right)}{\left(1-p^{i d+d_{i}} T^{d+i-t}\right)\left(1-p^{d i+n_{i}} T^{d+i}\right)}
\end{aligned}
$$

where $t=2$ in the case of points and $t=1$ in the case of lines and higher dimensional Fano varieties.

To prove the proposition, we use the following crucial lemma.

\section{Lemma 4.13.}

$$
\begin{aligned}
& \sum_{r_{i}=1}^{\infty} \sum_{b_{1}=1}^{r_{i}} \sum_{b_{2}=1}^{r_{i}} \cdots \sum_{b_{c_{i}}=1}^{r_{i}} \mu\left(r_{i} ; b_{1}, b_{2}, \ldots, b_{c_{i}}\right) p^{\left(i d+d_{i}\right) r_{i}} T^{(d+i) r_{i}} T^{-t \min \left\{a, b_{1}, b_{2}, \ldots, b_{c_{i}}\right\}} \\
& =\frac{p^{i d+d_{i}} T^{d+i-t}\left(1-p^{i d+d_{i}} T^{d+i}\right)}{\left(1-p^{i d+d_{i}} T^{d+i-t}\right)\left(1-p^{i d+d_{i}+c_{i}} T^{d+i}\right)}
\end{aligned}
$$

Now we can prove the proposition.

Proof. (of proposition 4.12)

$$
\begin{aligned}
A_{i^{*}}-A_{i} & =\sum_{r_{i}=1}^{\infty} \sum_{b_{1}=1}^{r_{i}} \sum_{b_{2}=1}^{r_{i}} \ldots \sum_{b_{n_{i}}=1}^{r_{i}} \mu\left(r_{i} ; b_{1}, b_{2}, \ldots, b_{n}\right) p^{i d r_{i}} T^{(d+i) r_{i}} T^{-t \min \left\{r_{i}, b_{1}, b_{2}, \ldots, b_{c_{i}}\right\}} \\
& -\sum_{r_{i}=1}^{\infty} \sum_{b_{1}=1}^{r_{i}} \sum_{b_{2}=1}^{r_{i}} \cdots \sum_{b_{n_{i}}=1}^{r_{i}} \mu\left(r_{i} ; b_{1}, b_{2}, \ldots, b_{n}\right) p^{i d r_{i}} T^{(d+i) r_{i}}
\end{aligned}
$$

First we sum all the $b_{i}$ 's that don't appear in the min-expression using the properties of the $\mu$-function:

$$
\begin{aligned}
A_{i^{*}}-A_{i} & =p^{-d_{i}} \sum_{r_{i}=1}^{\infty} \sum_{b_{1}=1}^{r_{i}} \sum_{b_{2}=1}^{r_{i}} \cdots \sum_{b_{c_{i}}=1}^{r_{i}} \mu\left(r_{i}, b_{1}, b_{2}, \ldots, b_{c_{i}}\right) p^{\left(i d+d_{i}\right) r_{i}} T^{(d+i) r_{i}} T^{-t \min \left\{r_{i} ; b_{1}, b_{2}, \ldots, b_{c_{i}}\right\}} \\
& -p^{-\left(d_{i}+c_{i}\right)} \sum_{r_{i}=1}^{\infty} p^{\left(i d+d_{i}+c_{i}\right) r_{i}} T^{(d+i) r_{i}}
\end{aligned}
$$


Now using lemma 4.13 and summation of geometric progressions we get

$$
\begin{aligned}
A_{i^{*}}-A_{i} & =p^{-d_{i}} \frac{p^{i d+d_{i}} T^{d+i-t}\left(1-p^{i d+d_{i}} T^{d+i}\right)}{\left(1-p^{i d+d_{i}} T^{d+i-t}\right)\left(1-p^{i d+d_{i}+c_{i}} T^{d+i}\right)} \\
& -p^{-\left(d_{i}+c_{i}\right)} \frac{p^{i d+d_{i}+c_{i}}}{1-p^{i d+c_{i}+d_{i}} T^{d+i}},
\end{aligned}
$$

and by a routine calculation we get the formula given in the corollary.

And finally, the proof of lemma 4.13 depends on the following combinatorial observations:

\section{Lemma 4.14.}

$$
\begin{aligned}
& \sum_{b_{1}=1}^{r_{i}+1} \sum_{b_{2}=1}^{r_{i}+1} \cdots \sum_{b_{c_{i}}=1}^{r_{i}+1} \mu\left(r_{i}+1 ; b_{1}, b_{2}, \ldots, b_{c_{i}}\right) T^{-t \min \left\{r_{i}+1, b_{1}, b_{2}, \ldots, b_{c_{i}}\right\}} \\
& =T^{-t} \sum_{b_{1}=1}^{r_{i}} \sum_{b_{2}=1}^{r_{i}} \cdots \sum_{b_{c_{i}}=1}^{r_{i}} \mu\left(r_{i} ; b_{1}, b_{2}, \ldots, b_{c_{i}}\right) T^{-t \min \left\{r_{i}, b_{1}, b_{2}, \ldots, b_{c_{i}}\right\}}+T^{-t} p^{c_{i} r_{i}}\left(1-p^{-c_{i}}\right)
\end{aligned}
$$

\section{Lemma 4.15.}

$$
p^{n k}\left(1-p^{-n}\right)=\left(\begin{array}{c}
n \\
1
\end{array}\right) p^{k}\left(1-p^{-1}\right)\left(p^{k-1}\right)^{n-1}+\left(\begin{array}{c}
n \\
2
\end{array}\right)\left(p^{k}\left(1-p^{-1}\right)\right)^{2}\left(p^{k-1}\right)^{n-2}+\cdots+\left(p^{k}\left(1-p^{-1}\right)\right)^{n}
$$

Proof. Binomial theorem.

\section{Lemma 4.16.}

$$
\begin{aligned}
& \sum_{b_{1}=2}^{r_{i}+1} \sum_{b_{2}=2}^{r_{i}+1} \cdots \sum_{b_{c_{i}}=2}^{r_{i}+1} \mu\left(r_{i}+1 ; b_{1}, b_{2}, \ldots, b_{c_{i}}\right) T^{-t \min \left\{r_{i}+1, b_{1}, b_{2}, \ldots, b_{c_{i}}\right\}} \\
& =T^{-t} \sum_{b_{1}=1}^{r_{i}} \sum_{b_{2}=1}^{r_{i}} \cdots \sum_{b_{c_{i}}=1}^{r_{i}} \mu\left(r_{i} ; b_{1}, b_{2}, \ldots, b_{c_{i}}\right) T^{-t \min \left\{r_{i}, b_{1}, b_{2}, \ldots, b_{c_{i}}\right\}}
\end{aligned}
$$

Proof. Change the range of summation and observe that $\mu$ is invariant under this action.

Next we prove lemma 4.14. 
Proof. We split the range of summation into two parts according to whether the min-expression takes value equal or greater than 1 . Then

$$
\begin{aligned}
& \sum_{b_{1}=1}^{r_{i}+1} \cdots \sum_{b_{c_{i}}=1}^{r_{i}+1} \mu\left(r_{i}+1 ; b_{1}, \ldots, b_{c_{i}}\right) T^{-t \min \left\{r_{i}+1, b_{1}, \ldots, b_{c_{i}}\right\}}= \\
& =T^{-t}(\text { number of terms for which } \min \text { is }=1)+ \\
& +\sum_{b_{1}=2}^{r_{i}+1} \cdots \sum_{b_{c_{i}}=2}^{r_{i}+1} \mu\left(r_{i}+1 ; b_{1}, \ldots, b_{c_{i}}\right) T^{-t \min \left\{r_{i}+1, b_{1} \ldots, b_{c_{i}}\right\}} \\
& =T^{-t} \cdot(\text { no of }\{\min =1\})+T^{-t} \sum_{b_{1}=1}^{r_{i}} \cdots \sum_{b_{c_{i}}=1}^{r_{i}} \mu\left(r_{i} ;, b_{1}, \ldots, b_{c_{i}}\right) T^{-t \min \left\{r_{i}, b_{1}, \ldots, b_{c_{i}}\right\}}
\end{aligned}
$$

by lemma 4.16. Now all that is left is to count the terms for which $\min =1$. As all the $b_{i}$ are integers between 1 and $r_{i}+1$, the only way that $\min \left\{r_{i}+\right.$ $\left.1, b_{1}, \ldots, b_{c_{i}}\right\}=1$ is when one or more of the $b_{i}=1$. Note that $\mu\left(r_{i}+1,1\right)=$ $p^{r_{i}}\left(1-p^{-1}\right)$, and $\sum_{b_{i}=2}^{r_{i}+1} \mu\left(r_{i}+1, b_{i}\right)=p^{r_{i}-1}$, so with these we have multiplicity $\left(\begin{array}{c}c_{i} \\ r\end{array}\right)\left(p^{r}\left(1-p^{-1}\right)\right)^{m}\left(p^{r_{i}-1}\right)^{c_{i}-m}$ when exactly $m$ of the $b_{i}$ are equal to one. Now putting these together and using lemma 4.15 we have the multiplicity of $T^{-t}$ $p^{c_{i} r_{i}}\left(1-p^{-c_{i}}\right)$ as claimed. This proves lemma 4.14.

And then finally lemma 4.13 .

Proof. (of lemma 4.13) Write

$$
A_{i^{*}}^{\prime}=\sum_{r_{i}=1}^{\infty} p^{\left(i d+d_{i}\right) r_{i}} T^{(d+i) r_{i}} \sum_{b_{1}=1}^{r_{i}} \sum_{b_{2}=1}^{r_{i}} \cdots \sum_{b_{c_{i}}=1}^{r_{i}} \mu\left(r_{i} ; b_{1}, b_{2}, \ldots, b_{c_{i}}\right) T^{-t \min \left\{r_{i}, b_{1}, b_{2}, \ldots, b_{c_{i}}\right\}}
$$

We split $A_{i^{*}}^{\prime}$ into pieces when $r_{i}=1$ and $r_{i} \geq 2$ and in the latter we change the variable $r_{i}=k+1$.

$$
\begin{aligned}
A_{i^{*}}^{\prime} & =p^{i d+d_{i}} T^{d+i-t}+ \\
& +p^{i d+d_{i}} T^{d+i} \sum_{k=1}^{\infty} p^{\left(i d+d_{i}\right) k} T^{(d+i) k} \sum_{b_{1}=1}^{k+1} \cdots \sum_{b_{c_{i}}=1}^{k+1} \mu\left(k+1 ; b_{1}, b_{2}, \ldots, b_{c_{i}}\right) T^{-t \min \left\{k+1, b_{1}, b_{2}, \ldots, b_{c_{i}}\right\}} \\
& =p^{i d+d_{i}} T^{d+i-t}+ \\
& +p^{i d+d_{i}} T^{d+i-t} \sum_{k=1}^{\infty} p^{\left(i d+d_{i}\right) k} T^{(d+i) k} \sum_{b_{1}=1}^{k} \cdots \sum_{b_{c_{i}}=1}^{k} \mu\left(k, b_{1}, b_{2}, \ldots, b_{c_{i}}\right) T^{-t \min \left\{k ; b_{1}, b_{2}, \ldots, b_{c_{i}}\right\}} \\
& +\left(1-p^{-c_{i}}\right) p^{i d+d_{i}} T^{d+i-t} \sum_{k=1}^{\infty} p^{\left(i d+d_{i}+c_{i}\right) k} T^{(d+i) k}
\end{aligned}
$$


using lemma 4.14 Now

$$
\begin{aligned}
\left(1-p^{\left(i d+d_{i}\right)} T^{d+i-t}\right) A_{i^{*}}^{\prime} & =p^{i d+d_{i}} T^{d+i-t}+\left(1-p^{-c_{i}}\right) p^{i d+d_{i}} T^{d+i-t} \sum_{k=1}^{\infty} p^{\left(i d+d_{i}+c_{i}\right) k} T^{(d+i) k} \\
& =p^{i d+d_{i}} T^{d+i-t}+\left(1-p^{-c_{i}}\right) p^{i d+d_{i}} T^{d+i-t} \frac{p^{i d+d_{i}+c_{i}} T^{d+i}}{1-p^{i d+d_{i}+c_{i}} T^{d+i}}
\end{aligned}
$$

just summing up the geometric progressions. Now rearranging our rational functions we get

$$
A_{i^{*}}^{\prime}=\frac{p^{i d+d_{i}} T^{d+i-t}\left(1-p^{i d+d_{i}} T^{d+i}\right)}{\left(1-p^{i d+d_{i}} T^{d+i-t}\right)\left(1-p^{i d+d_{i}+c_{i}} T^{d+i}\right)}
$$

as claimed.

\section{$5 \quad$ Functional equation}

The functional equation in this example is now an easy corollary of the general shape of the zeta function. It is conjectured in [4] Conjecture 5.47 that all uniform zeta functions of class two nilpotent groups satisfy the functional equation

$$
\left.\zeta_{G, p}^{\triangleleft}(s)\right|_{p \mapsto p^{-1}}=(-1)^{d+d^{\prime}} p^{\left(\begin{array}{c}
d+d^{\prime} \\
2
\end{array}\right)-\left(2 d+d^{\prime}\right) s} \cdot \zeta_{G, p}^{\triangleleft}(s) .
$$

Recall from [12] the observation that

$$
\begin{gathered}
\left.\zeta_{G, p}^{\triangleleft}(s)\right|_{p \mapsto p^{-1}}=(-1)^{d+d^{\prime}} p^{\left(\begin{array}{c}
d+d^{\prime} \\
2
\end{array}\right)-\left(2 d+d^{\prime}\right) s} \cdot \zeta_{G, p}^{\triangleleft}(s) \Leftrightarrow \\
\left.A(p, T)\right|_{p \mapsto p^{-1}}=(-1)^{d^{\prime}-1} p^{\left(\begin{array}{c}
d^{\prime} \\
2
\end{array}\right)} \cdot A(p, T) .
\end{gathered}
$$

We can also observe that in our decomposition of

$$
A(p, T)=W_{0}(p, T)+\sum_{i=1}^{3} \mathfrak{n}_{i}(p) W_{i}(p, T)
$$

it is enough to show that each of the summands satisfies the same functional equation as $A(p, T)$ satisfies. In this example $d=4$ and $d^{\prime}=6$, but we do this in general, since the functional equation doesn't depend on the actual numerical values $d$ and $d^{\prime}$ take.

It is known at least from [13] and can in fact be explicitly observed that

$$
\left.I_{k}(\mathbf{U})\right|_{U_{i} \mapsto U_{i}^{-1}}=(-1)^{k} p^{\left(\begin{array}{c}
k+1 \\
2
\end{array}\right)} I_{k}(\mathbf{U}),
$$


where $U_{i}=p^{a_{i}-b_{i} s}$.

So in each of

$$
W_{i}(X, Y)=I_{d^{\prime}-i-1}\left(X_{d^{\prime}-1}, \ldots, X_{i+1}\right) E_{i}\left(X_{i}, Y_{i}\right) I_{i-1}\left(Y_{i-1}, \ldots, Y_{1}\right)
$$

we have a functional equations from the Igusa factors as $(-1)^{d^{\prime}-i-1} p^{\left(\begin{array}{c}d^{\prime}-i \\ 2\end{array}\right)}$ and $(-1)^{i-1} p^{\left(\begin{array}{c}i \\ 2\end{array}\right)}$, and it is left to see what is the functional equation on $E_{i}$. But this is now easy since we have the explicit formula

$$
E_{i}\left(X_{i}, Y_{i}\right)=\frac{p^{-d_{i}} Y_{i}-p^{-n_{i}} X_{i}}{\left(1-X_{i}\right)\left(1-Y_{i}\right)}
$$

and we see that

$$
\left.E_{i}\left(X_{i}, Y_{i}\right)\right|_{\mapsto p^{-1}}=p^{n_{i}+d_{i}} E_{i}\left(X_{i}, Y_{i}\right) .
$$

Here $n_{i}=i\left(d^{\prime}-i\right)$ and $d_{i}=$ the dimension of the Fano variety of $(i-1)$ dimensional linear subspaces on $\mathfrak{P}$.

If we know observe that for $i=1,2,3$ the points on the Fano varieties have functional equations $\left.\mathfrak{n}_{i}(p)\right|_{p \mapsto p^{-1}}=p^{-d_{i}} \mathfrak{n}_{i}(p)$, since these $\mathfrak{n}_{i}(p)$ are polynomials in $p$ with nice symmetry properties.

So putting all of these together

$$
\left.W_{i}(p, T)\right|_{p \mapsto p^{-1}}=(-1)^{d^{\prime}-i-1+(i-1)} p^{\left(\begin{array}{c}
d^{\prime}-i \\
2
\end{array}\right)+n_{i}+\left(\begin{array}{c}
i \\
2
\end{array}\right)} W_{i}(p, T),
$$

and as $\left(\begin{array}{c}d^{\prime}-i \\ 2\end{array}\right)+n_{i}+\left(\begin{array}{c}i \\ 2\end{array}\right)=\left(\begin{array}{c}d^{\prime} \\ 2\end{array}\right)$ we have that

$$
\left.W_{i}(p, T)\right|_{p \mapsto p^{-1}}=(-1)^{d^{\prime}} p^{\left(\begin{array}{c}
d^{\prime} \\
2
\end{array}\right)} W_{i}(p, T)
$$

as required. This proves the functional equation.

\section{References}

[1] M.P.F. du Sautoy, A nilpotent group and its elliptic curve: nonuniformity of local zeta functions of groups, Israel J. of Math. 126 (2001), $269-288$.

[2] M.P.F. du Sautoy, Counting finite $p$-groups and nilpotent groups, Inst. Hautes Études Scientifiques, Publ. Math., 92 (2000), 63 - 112.

[3] M.P.F. du Sautoy, Counting subgroups in nilpotent groups and points on elliptic curves, J. Reine. Angew. Math. 549 (2002), 1 - 21. 
[4] M.P.F. du Sautoy, Zeta functions of groups: The quest for order versus the flight from ennui, Groups St Andrews 2001 - in Oxford, Volume 1, CUP 2003.

[5] M.P.F. du Sautoy and F.J. Grunewald, Analytic properties of zeta functions and subgroup growth, Annals of Math. 152 (2000), $793-833$.

[6] F.J. Grunewald, D. Segal and G.C. Smith, Subgroups of finite index in nilpotent groups, Invent. Math. 93 (1988), 185 - 223.

[7] J. Harris, Algebraic Geometry: A First Course, Springer-Verlag, New York, 1992.

[8] J.W.P. Hirschfeld and J.A. Thas, General Galois Geometries, Clarendon Press, Oxford, 1991.

[9] P.M. Paajanen, On the degree of polynomial subgroup growth in class 2 nilpotent groups, preprint.

[10] P.M. Paajanen, Structure and functional equations of normal zeta functions of class two nilpotent groups, in preparation.

[11] P.M. Paajanen, The Segre example - lines on Pfaffian, a chapter of DPhil thesis, Oxford, in preparation.

[12] C. Voll, Zeta functions of groups and enumeration in Bruhat-Tits buildings, Amer. J. Math. 126 (2004), no. 5, 1005 - 1032.

[13] C. Voll, Functional equations for local normal zeta functions of nilpotent groups, Geom. Funct. Anal., to appear. 